\title{
A Novel Vibration-Based Fault Diagnostic Algorithm for Gearboxes \\ Under Speed Fluctuations Without Rotational Speed Measurement
}

\author{
Liu Hong ${ }^{\text {a }}$ Yongzhi Qu ${ }^{\text {a }}$, Jaspreet Singh Dhupia ${ }^{*}, \mathrm{~b}$, Shuangwen Sheng ${ }^{\mathrm{c}}$, Yuegang Tan ${ }^{\mathrm{a}}$, Zude Zhou ${ }^{\mathrm{a}}$ \\ ${ }^{a}$ School of Mechanical and Electronic Engineering, Wuhan University of Technology, China \\ ${ }^{\mathrm{b}}$ Department of Mechanical Engineering, University of Auckland, New Zealand \\ ${ }^{\mathrm{c}}$ National Renewable Energy Laboratory, United States
}

\begin{abstract}
The localized failures of gears introduce cyclic-transient impulses in the measured gearbox vibration signals. These impulses are usually identified from the sidebands around gear-mesh harmonics through the spectral analysis of cyclo-stationary signals. However, in practice, several high-powered applications of gearboxes like wind turbines are intrinsically characterized by nonstationary processes that blur the measured vibration spectra of a gearbox and deteriorate the efficacy of spectral diagnostic methods. Although order-tracking techniques have been proposed to improve the performance of spectral diagnosis for nonstationary signals measured in such applications, the required hardware for the measurement of rotational speed of these machines is often unavailable in industrial settings. Moreover, existing tacho-less order-tracking approaches are usually limited by the high time-frequency resolution requirement, which is a prerequisite for the precise estimation of the instantaneous frequency. To address such issues, a novel fault-signature enhancement algorithm is proposed that can alleviate the spectral smearing without the need of rotational speed measurement. This proposed tacho-less diagnostic technique resamples the measured acceleration signal of the gearbox based on the optimal warping path evaluated from the fast dynamic time-warping algorithm, which aligns a filtered shaft rotational harmonic signal with respect to a reference signal assuming a constant shaft rotational speed estimated from the approximation of operational speed. The effectiveness of this method is validated using both simulated signals from a fixed-axis gear pair under nonstationary conditions and experimental measurements from a $750-\mathrm{kW}$ planetary wind turbine gearbox on a dynamometer test rig. The results
\end{abstract}


demonstrate that the proposed algorithm can identify fault information from typical gearbox vibration measurements carried out in a resource-constrained industrial environment.

Keywords: gear fault diagnosis; tacho-less algorithm; speed fluctuations

\section{Introduction}

The characteristic frequencies of gear faults in the measured vibration spectrum are harmonically related to the shaft rotational speed. A localized gear failure results in periodic amplitude and phase modulations in which spectral sidebands are spaced around the gear-mesh harmonics at a distance equal to the shaft rotational harmonics $[1,2]$. Assuming a constant shaft rotational frequency, the sidebands can be easily identified in theory through the spectral analysis of cyclo-stationary signals $[3,4]$. However, in practice, though rotating machines can be operated at a relatively stationary condition, the gearboxes - especially those employed in high-powered applications - are often characterized by significant speed fluctuations. For instance, Ref. [5] presents the rotational speed during the controlled operation of an $850-\mathrm{kW}$ variable-speed wind turbine drivetrain in which $18 \%$ to $32 \%$ speed variations with respect to the nominal operational speed can be observed within 85 s. Such speed fluctuations can smear the frequency components in the measured vibration spectrum, which makes it difficult to recognize the sidebands introduced by a gear fault.

This spectral smearing phenomenon occurring as a result of speed fluctuations can be alleviated by order-tracking (OT) methods. Conventional OT techniques employ additional sensors to provide the information of rotational speed or angular position of the shaft, which increase costs and are often unavailable in industrial applications [6]. The first tacho-less OT algorithm for gear diagnosis was presented in [7], which estimated the shaft angular position using a narrow-band phase demodulation at one of the harmonics of the gear-mesh frequency. The required harmonic of the gear-mesh frequency was extracted by an appropriately designed band-pass filter. This algorithm was further mathematically investigated in [8]. It was proven that the gear-mesh harmonics (the higher-order harmonics of shaft rotational frequency), instead of the 
shaft rotational frequency or any of its low harmonics, should be extracted to conduct the phase demodulation for lowering the estimated shaft phase error. However, the use of a high-order harmonic of shaft rotational frequency (i.e., gear-mesh harmonics) restricts the maximum allowed speed fluctuation of the algorithm (typically to less than $1 \%)[7,8]$. To compensate for larger speed variations, the time frequency distribution (TFD) algorithms that evaluate the instantaneous frequency (IF) of gear-mesh harmonics on the basis of ridge searching of TFD have attracted significant attention recently [9-11]. Though the TFD-based methods are also capable of estimating the rotational speed of the shaft, they are usually limited by a high time-frequency resolution requirement in practice, which is a prerequisite for the precise estimation of IF [12]. Moreover, several gear-mesh harmonics are not even present in the vibration spectrum of an equally spaced sequential planetary gearbox $[13,14]$. Generally, these issues deteriorate the efficacy of the existing tacho-less OT methods during their applications in an industrial environment.

To address such aforementioned issues and advance the state of the art of tacho-less diagnostic techniques, a novel fault-diagnostic algorithm for gearboxes under speed fluctuations is developed in this paper. The proposed approach alleviates the problem of spectral smearing by resampling the measured acceleration signal of the gearbox based on the optimal warping path evaluated from the fast dynamic time warping (Fast DTW) algorithm, which dynamically aligns a filtered shaft rotational harmonic with a reference signal assuming a constant shaft rotational speed. The reference signal is chosen as a simple monocomponent sinusoidal function in which frequency and amplitude are estimated from the approximated operational speed and vibration characteristics of either the input or output shaft. Thus, the presented diagnostic method requires only an approximate knowledge of the operational speed besides the measured gearbox vibration signal. The capability of the approach is first demonstrated through a simulation study of a fixed-axis gear pair under nonstationary conditions. Later, the effectiveness of this approach is also experimentally validated through the measured vibration signal from a commercial $750-\mathrm{kW}$ planetary wind turbine gearbox. 
The rest of the paper is organized as follows. Section 2 gives a brief background of the Fast DTW algorithm. The detailed procedure for the proposed tacho-less fault diagnostic technique is described and discussed in Section 3. Section 4 investigates the effectiveness of the proposed method using MATLAB simulation studies. Section 5 presents the experimental validation of a $750-\mathrm{kW}$ planetary gearbox that was damaged during the field operation while installed in a wind turbine. Finally, Section 6 concludes the paper.

\section{Review of fast dynamic time warping}

The proposed fault diagnostic procedure for gearboxes proposed in this paper alleviates the effect of spectral smearing due to speed fluctuations by resampling the data based on the optimal warping path evaluated from the Fast DTW algorithm. This approach demonstrates a promising performance to identify fault signatures using captured vibration data without any rotational speed measurement. DTW and Fast DTW are reviewed briefly in this section to provide greater understanding.

\subsection{Dynamic time-warping algorithm}

A simple compression or expansion along the timescale is not enough to accurately align two similar time-domain vibration signals from practical rotating machineries, which differ because of the random nonlinear fluctuations in speed. DTW can find the optimal alignment between the corresponding data points from the two series yielding minimum residue, which allows the timescale to be warped nonlinearly by stretching or shrinking. Thus, DTW is a useful tool to determine the similarity between two given time series [15]. It was recently employed to detect faults in a reciprocating compressor using driving motor current signals in [16], which assumed the reference current signal under healthy operating conditions to be sinusoidal at line frequency and the similarity between the measured current signal and the reference sinusoidal signal will decrease in the presence of a fault.

Figure 1 illustrates the process of DTW, and the problem can be formally stated as follows: given two time series, $X$ and $Y$ of length $N$ and $M$, respectively.

$$
X=x_{1}, x_{2}, \ldots, x_{i}, \ldots, x_{N}
$$



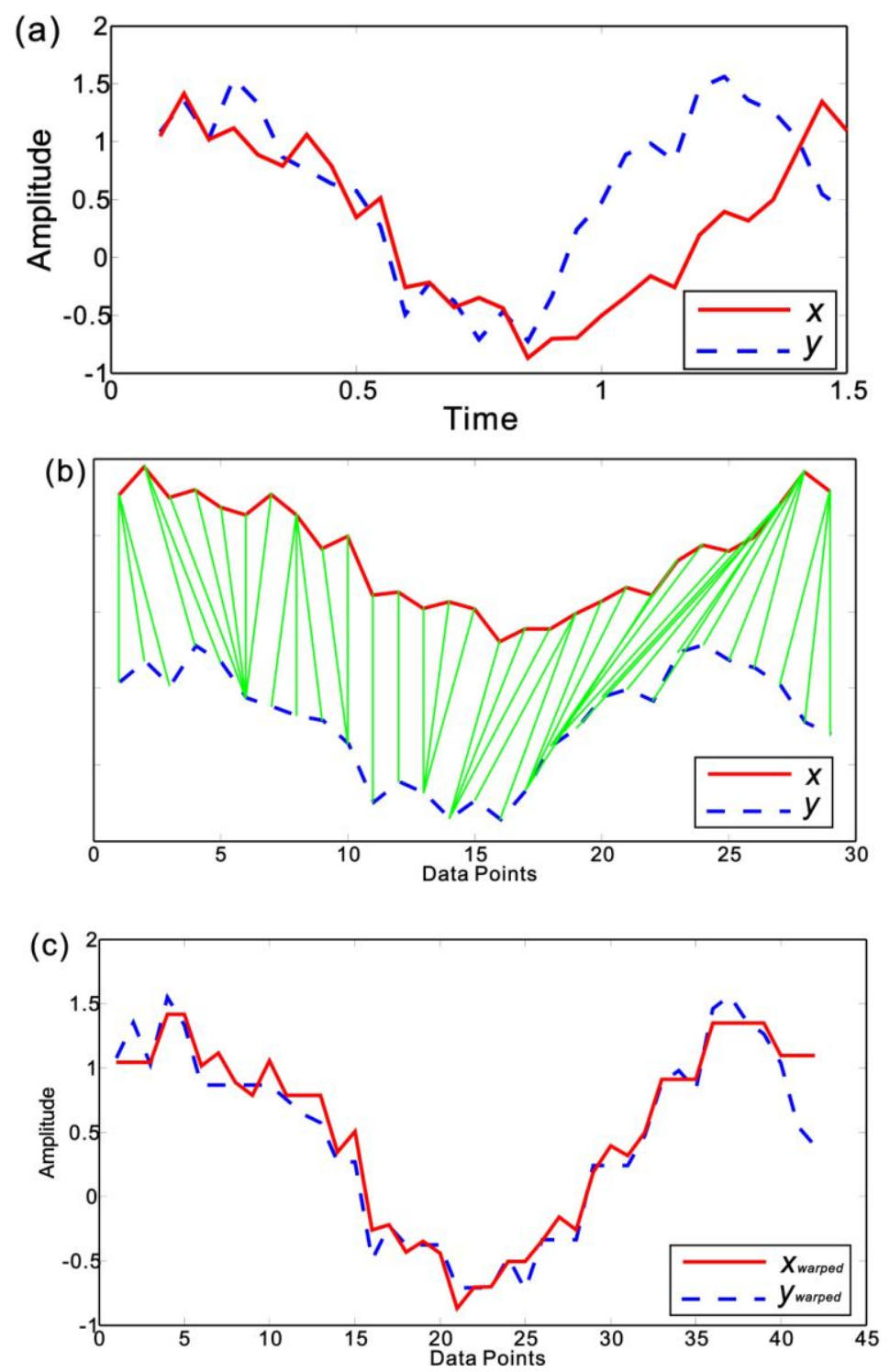

Fig. 1. Dynamic time warping (DTW) applied to a pair of time series (a) before, (b) the corresponding similar value points found by DTW, and (c) after the

$$
Y=y_{1}, y_{2}, \ldots, y_{i}, \ldots, y_{M}
$$

Construct a warping path, $W$ :

$$
W=w_{1}, w_{2}, \ldots, w_{k}, \ldots, w_{K}
$$

$K$ is the length of the warping path and the $k$ th element of the warping path is:

$$
w_{k}=(i, j)
$$

where $i\left(=w_{k}(1,1)\right)$ is an index from the time series, $X$, and $j\left(=w_{k}(1,2)\right)$ is an index from the time series, $Y$. If the warping path, $W$, contains the element, $(i, j)$, as in Eq. (4), it implies that the $i$-th sample of the time series, $X$, is aligned with the $j$-th 


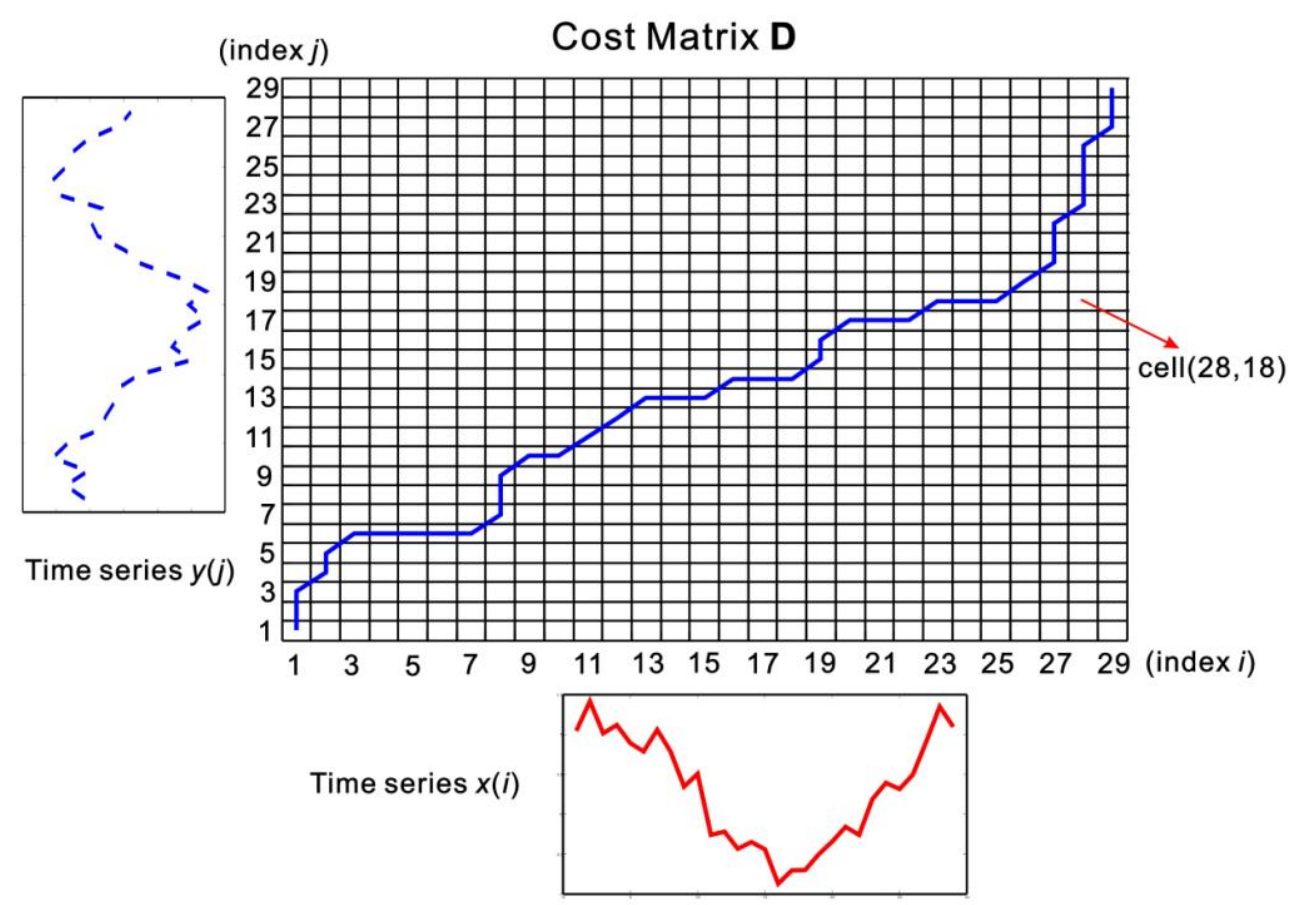

Fig. 2. The cost matrix, D, of DTW indicating the optimal alignment path for the time series, $X$ and $Y$, presented in Fig. 1.

sample of the time series, $Y$. Moreover, the warping path must satisfy the following conditions $[15,17]$ :

a) $w_{1}=(1,1)$, which implies that the warping path must start at the beginning of each time series,

b) $w_{K}=(N, M)$, which implies that the warping path must finish at the end of both time series, and

c) if $w_{k}=(i, j)$ and $w_{k+1}=\left(i^{\prime}, j^{\prime}\right)$, then $i^{\prime} \in(i, i+1)$ and $j^{\prime} \in(j, j+1)$, which implies that every index in both time series must be utilized at least once and sequentially between the start and end of the warping path.

To evaluate this warping path, a two-dimensional cost matrix, D, (the accumulative distance matrix, typically Euclidean distance) of dimension, $N \times M$, (Fig. 2) is calculated, in which a cell, $\mathbf{D}(i, j)=(x(i)-y(j))^{2}$. Afterwards, the optimal warping path can be found by using a dynamic programming approach, which minimizes the accumulative distance between the two time series. Because each cell in the cost matrix, D, is evaluated once, it makes the DTW algorithm quadratic in both time and 
space complexity.

2.2. Fast implementation of dynamic time warping

The $\mathrm{O}\left(N^{2}\right)$ time and space complexity of DTW constrains its usefulness only to the time series of length no more than a few thousand data points $[15,17]$. However, the vibration signals of gearboxes are often much longer because they are measured at a sampling frequency of several kilohertz. Thus, speeding up the computational time and reducing the memory requirement are crucial to implementing the DTW algorithm in most vibration-based fault-detection problems of gearboxes. With this motivation, a multilevel approach called Fast DTW, running in a linear $\mathrm{O}(N)$ time and space complexity, was employed for the residual signal analysis of gearboxes [18]. This involves a three-step recursive approach. First, a lower-level/resolution time series is created by averaging adjacent pairs of points of the input time series. Consequently, the resulting lower-level/resolution time series has half as many points as the input time series for a single call to the recursive function. Next, an optimal warping path in a lower level/resolution is found and projected to the next higher level/resolution. Finally, the projected path is expanded by a predefined radius to form a search window at the higher level/resolution that is passed to a constrained DTW algorithm. This constrained DTW only evaluates the cells within the search window to find the optimal warping path. The recursive function can be called several times during the implementation of the algorithm, which generates multiple

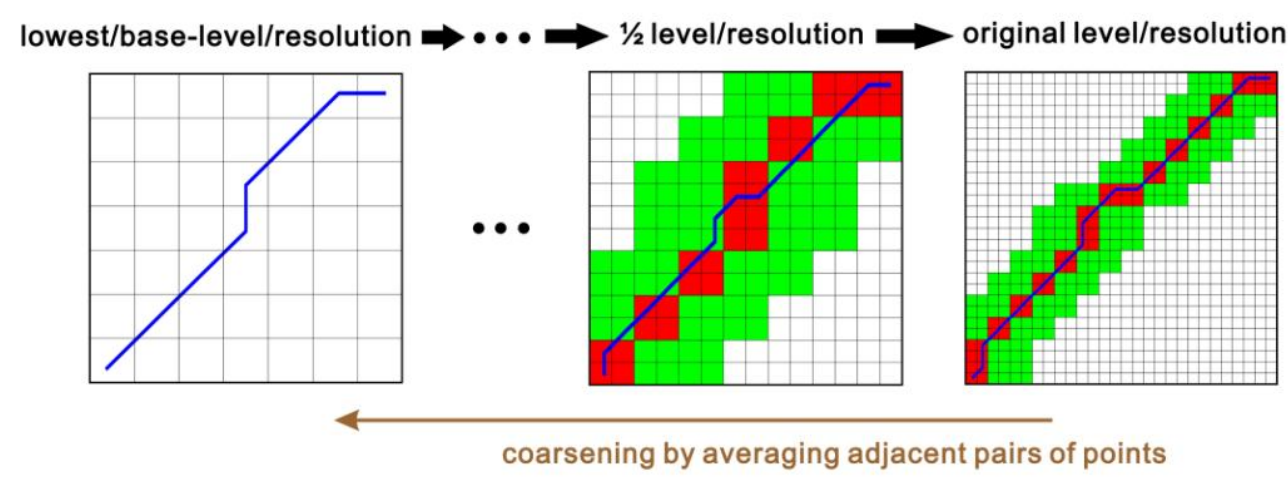

Fig. 3. The window and optimal path evaluated at different levels/resolutions in the recursive Fast DTW algorithm with a predefined radius of four cells. 
levels/resolutions until the lowest-level/resolution time series contains only a few hundred points. At each call of the recursive function, the warping path calculated in lower level/resolution is projected to its next higher level/resolution and the constrained DTW algorithm is used to evaluate the optimal warp path for that level. However, for the lowest-level/resolution time series, the standard DTW algorithm is directly employed to find the optimal warping path. Figure 3 graphically illustrates the idea of this three-step recursive approach of the Fast DTW algorithm. The optimal warping path evaluated using the DTW algorithm in the lowest level/resolution is shown as a blue line on the left-hand side of Fig. 3. This path is projected to the next higher level/resolution expanded by a predefined radius. The shaded cells in the figure indicate the search window at the higher level/resolution, in which the red cells represent the projection of the optimal path evaluated in the lower resolution, and the green cells represent the predefined radius. The constrained DTW only searches for the optimal warping path in the shaded region at a given level/resolution. Because the search windows have a constant thickness (or predefined radius), the number of evaluated cells at the end of recursive call are linearly related to length of time series, $N$. Therefore, this fast implementation reduces the time and space complexity of DTW. Generally, DTW/Fast DTW can align two similar signals that have a phase difference or speed fluctuations that are often encountered in practical rotating machineries [19].

\section{Scheme of the proposed tacho-less diagnostic algorithm}

Because the direct measurement of the rotational speed of rotating machineries is often unavailable, recent publications have shown interest in the diagnosis of gearboxes under the nonstationary conditions using only the measured vibration signals [7-12]. However, most of the existing tacho-less techniques are still constrained by complex vibration characteristics of multistage and/or planetary gearboxes under significant speed fluctuations and high time-frequency resolution requirements, which may challenge their implementation in industrial environments. To address such issues, a general vibration-based algorithm for the diagnosis of gearboxes without the use of any rotational speed measurement is proposed herein. 


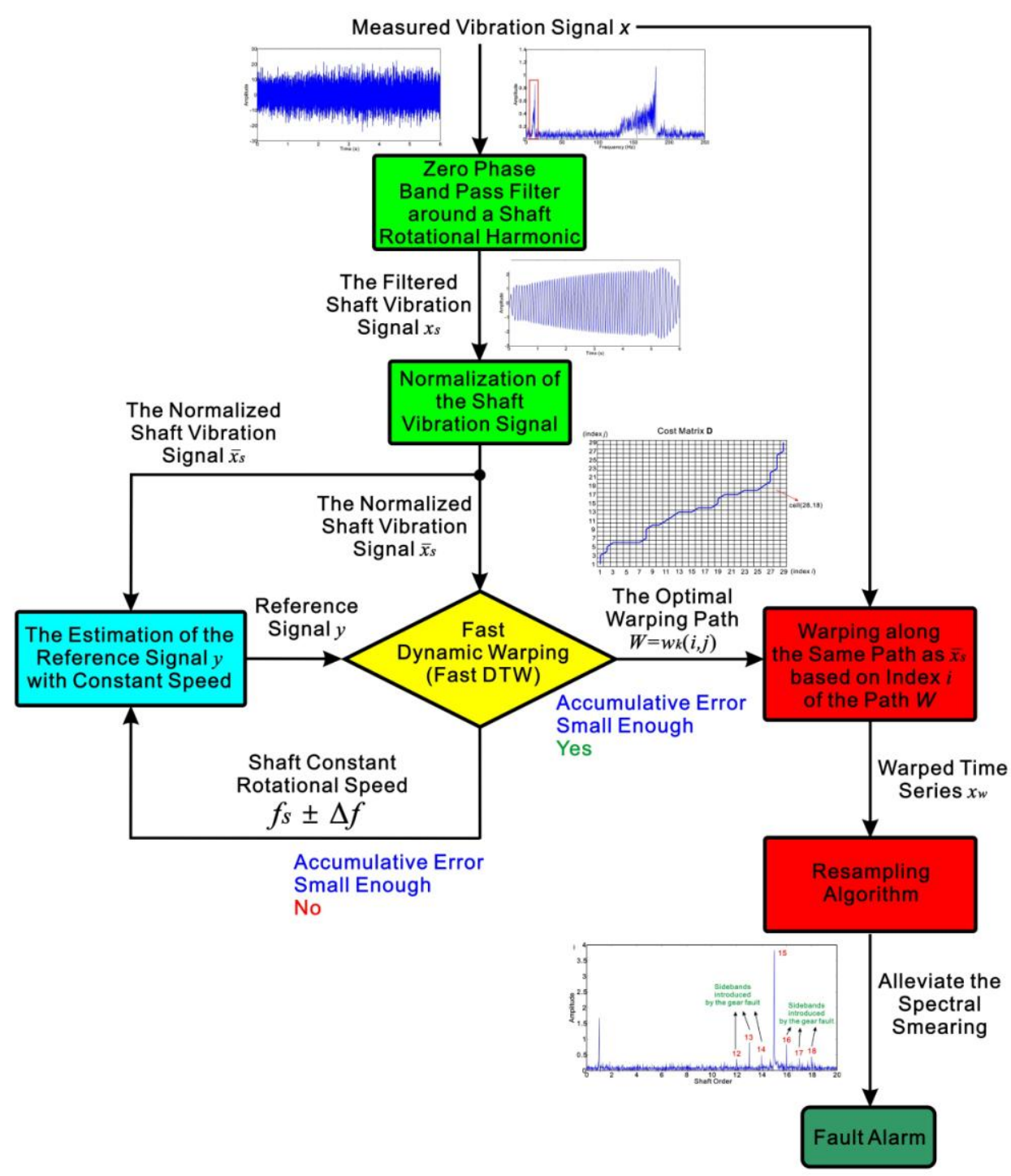

Fig. 4. Flowchart of the proposed tacho-less diagnostic algorithm.

The key steps of the developed diagnostic scheme are the extraction of a shaft rotational harmonic, the estimation of a reference signal with an assumed constant speed, the Fast DTW implementation to align the extracted shaft vibration signal with the reference signal, and the warping and resampling of the original vibration measurement to alleviate the spectral smearing caused by speed fluctuations. The overall presented scheme is summarized in Fig. 4 and the details are explained as follows:

Step 1: Extraction of a harmonic of the shaft rotational frequency

First of all, the captured original vibration signal, $x(t)$, is processed by a zero-phase band-pass filter to extract a monocomponent sinusoidal signal, the frequency of which 
corresponds to the $n$th harmonic of either the input or output shaft rotational speed $f_{s}(t)$. The bandwidth, $B$, of the filter must be designed to ensure that all the energy of the $n$th shaft rotational harmonic, $n f_{s}(t)$, falls within the passband of the filter even after accounting for the speed variations and approximated errors of the nominal operational speed. However, on the other hand, the bandwidth $B$ should not be chosen to be excessive, otherwise it introduces undesired noise in the extracted waveform. Moreover, the bandwidth, $B$, should not overlap with a lower or higher order of the shaft rotational harmonic. When dealing with a signal having a rapid ramp speed profile, such as during the start-up or run-down process, the Vold-Kalman filter can be employed instead of the traditional band-pass filter to avoid the overlapping issue [20, 21]. In practice, the first three harmonics $(n \leq 3)$ of the shaft rotational frequency, $f_{s}(t)$, are often observed in the low-frequency region of gearbox vibration spectra. Therefore, the most dominant harmonic among them can be extracted to yield a higher signal-to-noise ratio (SNR). The extracted shaft vibration signal $x_{s}(t)$ can be represented as:

$$
x_{s}(t)=A(t) \cos \left(2 \pi n f_{s}(t) t+\varphi\right)
$$

where $\varphi$ is the initial phase and $A(t)$ is the time-dependent amplitude of the extracted harmonic, which corresponds to the external load variation and the effect of filter distortion.

For the ease of constructing the reference signal in the next step, $x_{s}(t)$ is further demodulated to form a normalized sinusoidal function, $\bar{x}_{s}(t)$, using the Hilbert transform, which eliminates the amplitude variation caused by both the load variation

\section{Table 1}

The points used for curve fitting of the shaft rotational speed simulation.

\begin{tabular}{lccccccccc}
$\begin{array}{l}\text { No. of } \\
\text { Points }\end{array}$ & 1 & 2 & 3 & 4 & 5 & 6 & 7 & 8 & 9 \\
\hline $\begin{array}{l}\text { Time } \\
\text { Instant (s) }\end{array}$ & 0 & 0.2 & 0.4 & 0.6 & 0.8 & 1.0 & 1.2 & 1.4 & 1.6 \\
\hline $\begin{array}{l}\text { Frequency } \\
\text { Value } \\
(\mathbf{H z})\end{array}$ & 3.2 & 4 & 4.7 & 4.6 & 4.5 & 4.3 & 4.6 & 4.3 & 4.5 \\
\hline
\end{tabular}



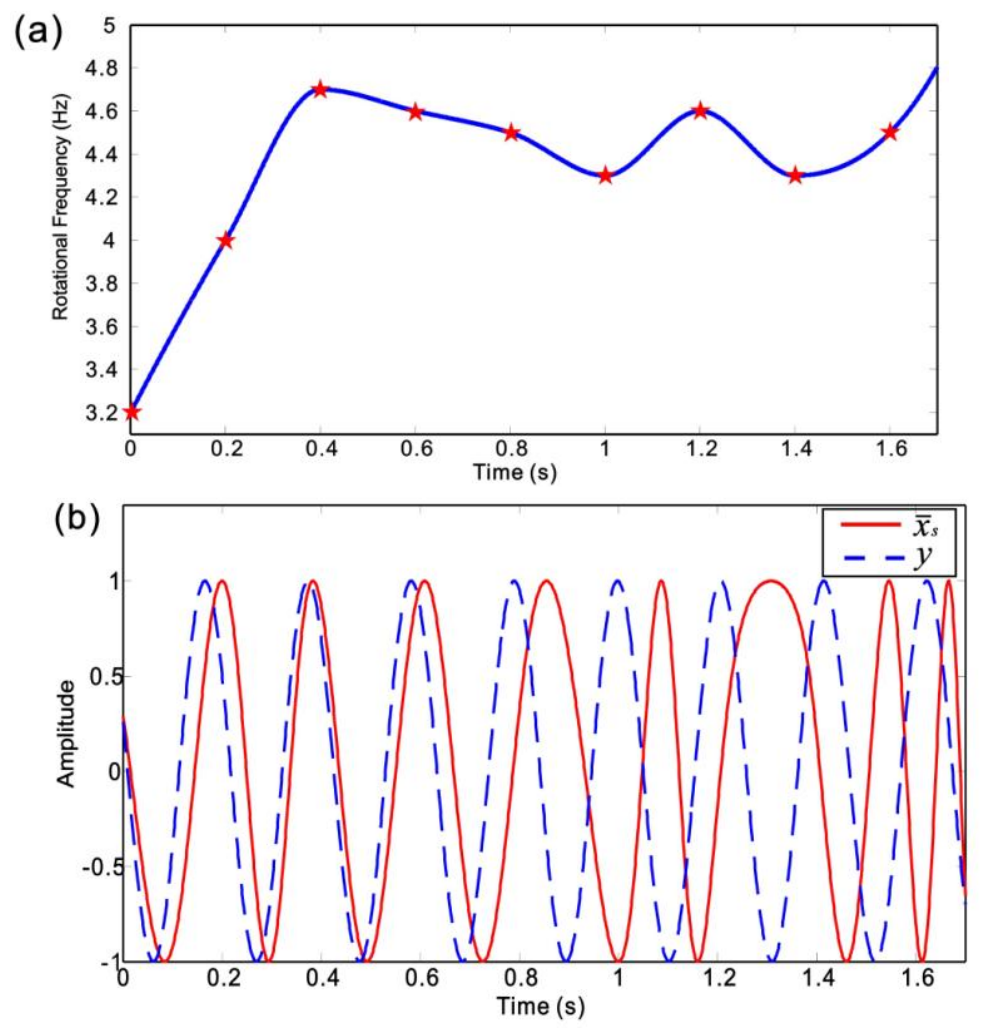

Fig. 5. (a) Time-dependent rotational speed $f_{s}(t)$ profile used for illustrating the algorithm, wherein red stars represent the points used for curve fitting, and (b) is the evaluated normalized function $\bar{x}_{s}(t)$ and the reference function $y(t)$ with a constant frequency $f_{s}$.

and the filter distortion:

$$
\begin{gathered}
A(t)=\left(\tilde{x}_{s}^{2}(t)+x_{s}^{2}(t)\right)^{\frac{1}{2}} \\
\bar{x}_{s}(t)=\frac{x_{s}(t)}{A(t)}=\cos \left(2 \pi n f_{s}(t) t+\varphi\right)
\end{gathered}
$$

where $\tilde{x}_{s}(t)$ is the Hilbert transform of $x_{s}(t)$. To illustrate the proposed diagnostic algorithm, Fig. 5(a) shows a rotational speed, $f_{s}(t)$, simulated by using a cubic spline interpolation to the nine points listed in Table 1, exhibiting a fluctuation of over $46 \%$ within 1.7 s. The initial phase $\varphi$, in Eq. (7), is arbitrarily chosen as $\frac{2 \pi}{5}$ and the corresponding simulated signal $\bar{x}_{s}(t)$ ( $n$ is set as 1), is plotted in Fig. 5(b) as the red solid line. The time interval, $\Delta t$, used in this illustration is $10^{-3}$ seconds. 


\section{Step 2: Estimation of the reference signal}

The estimated signal, $y(t)$, is built using the vibration characteristics of the reference shaft of the gearbox (either the input or output shaft can be chosen). If a gearbox is operated under a constant load and rotational speed, $f_{s}$, the vibration component of its $n$th shaft rotational harmonic can be represented as a simple sinusoidal signal $[12,22]$ :

$$
y(t)=A \cos \left(2 \pi n f_{s} t+\theta\right)
$$

where $A$ and $\theta$ are the amplitude and initial phase, respectively. This signal, $y(t)$, with a constant frequency, $f_{s}$, is used as the reference time series in the subsequent Fast DTW algorithm. $y(t)$ is built using the same sampling interval, $\Delta t$, and the total time, $T$, as the original vibration signal $x(t)$. According to Eq. (7), the amplitude of reference signal, $y(t)$, in Eq. (8) is set as $A=1$. Furthermore, the constant shaft rotational frequency, $f_{s}$, of the reference signal, $y(t)$, can be chosen from the range between $\frac{\left(P_{p}-1\right)}{n T}$ and $\frac{\left(P_{p}+1\right)}{n T} . P_{p}$ corresponds to the number of positive peaks of the sinusoidal signal, which can be estimated from the number of local maximum points of the normalized shaft-vibration signal $\bar{x}_{s}(t)$ (the local minimum points or zero-crossing points can also be used instead). If the estimation error of $f_{s}$ is found to affect the alignment between the reference, $y(t)$, and normalized shaft vibration signal $\bar{x}_{s}(t)$ after Step 3, such performance degradation can be minimized by sweeping $f_{s}$ within the determined range as shown in Fig. 4. The initial phase $\theta$ of the reference signal, $y(t)$, is matched with the normalized shaft vibration signal $\bar{x}_{s}(t)$ by setting as $\cos ^{-1}\left(\bar{x}_{s}(0)\right)$ if the gradient $\bar{x}_{s}(t)$ begins from a negative value $(0<\varphi \leq \pi)$; or else, $\theta$ equals $2 \pi-\cos ^{-1}\left(\bar{x}_{s}(0)\right)(\pi<\varphi \leq 2 \pi)$. The dashed blue line in Fig. 5(b) plots the corresponding time-domain waveform of the estimated reference signal, $y(t)$, with a constant frequency $f_{s}=4.8 \mathrm{~Hz}$, based on the simulated $\bar{x}_{s}(t)$ as the red solid line. Fig. 5(b) shows that the nonstationary normalized signal $\bar{x}_{s}(t)$ evaluated by Eq. (7), 


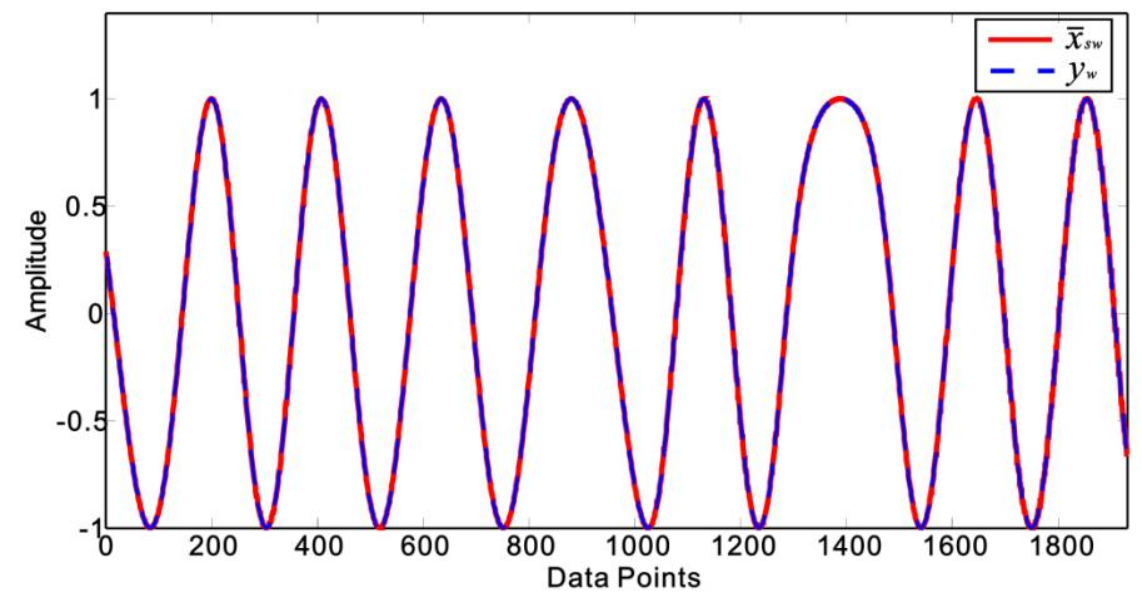

Fig. 6. The warped signals $\bar{x}_{s w}(k)$ and $y_{w}(k)$, of the signals $\bar{x}_{s}(t)$ and $y(t)$, are presented in Fig. 5(b) after the Fast DTW.

which includes the effect of speed fluctuations, appears like a nonlinear stretching/shrinking transformation of $y(t)$, evaluated by Eq. (8).

Step 3: Fast DTW implementation

The two signals, the normalized shaft vibration signal, $\bar{x}_{s}(t)$, and the estimated reference signal, $y(t)$, are matched in the time domain by employing the Fast DTW algorithm described in Section 2.2. The purpose of Fast DTW is to reveal the optimal alignment path, $W$, describing the best fit between the two signals. The phase or frequency differences from the effect of speed fluctuations are thus addressed by warping them along the timescale. As a result, the information regarding stretching/shrinking among the time instants caused by the speed fluctuations, $f_{s}(t)$, is also implicitly contained in the optimal alignment path, $W$. The warped signals after application of the Fast DTW are denoted as $\bar{x}_{s w}(k)$ and $y_{w}(k)$, respectively, and are shown in Fig. 6 . Note that the length $K$ of the warped signals, $\bar{x}_{s w}(k)$ and $y_{w}(k)$, are different from the length, $N$, of the original signals. The reason for this difference in length of data can be deduced from Fig. 2 and the constraint (c) of the warping path where it can be observed that the index $i$ ' or $j$ ' may equal $i$ or $j$, respectively, in the warping path function $w_{k}=(i, j), w_{k+1}=\left(i, j^{\prime}\right)$. Consequently, the temporal information is distorted along the horizontal axis of the warped signals $\bar{x}_{s w}(k)$ and 


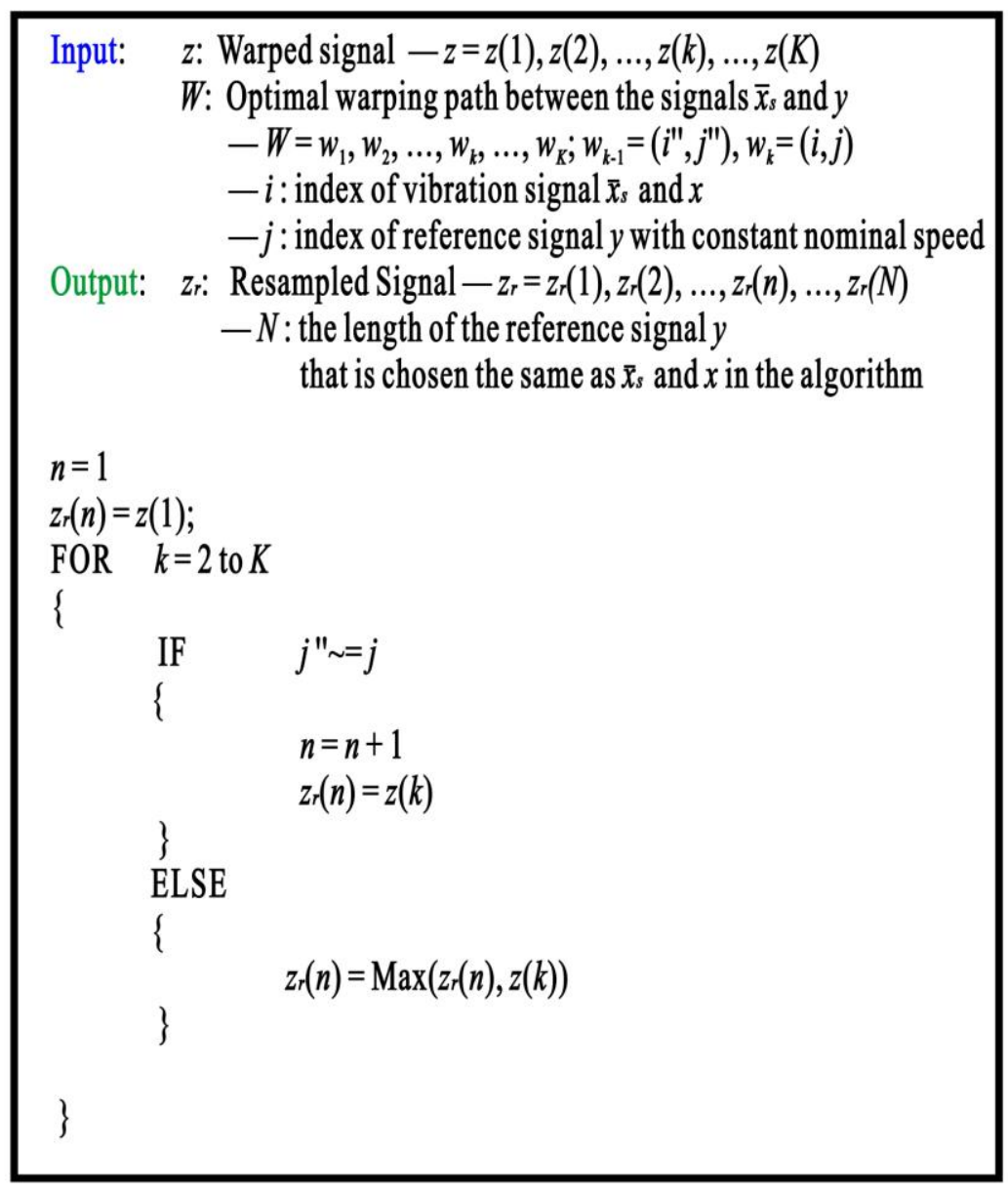

Fig. 7. Resampling algorithm.

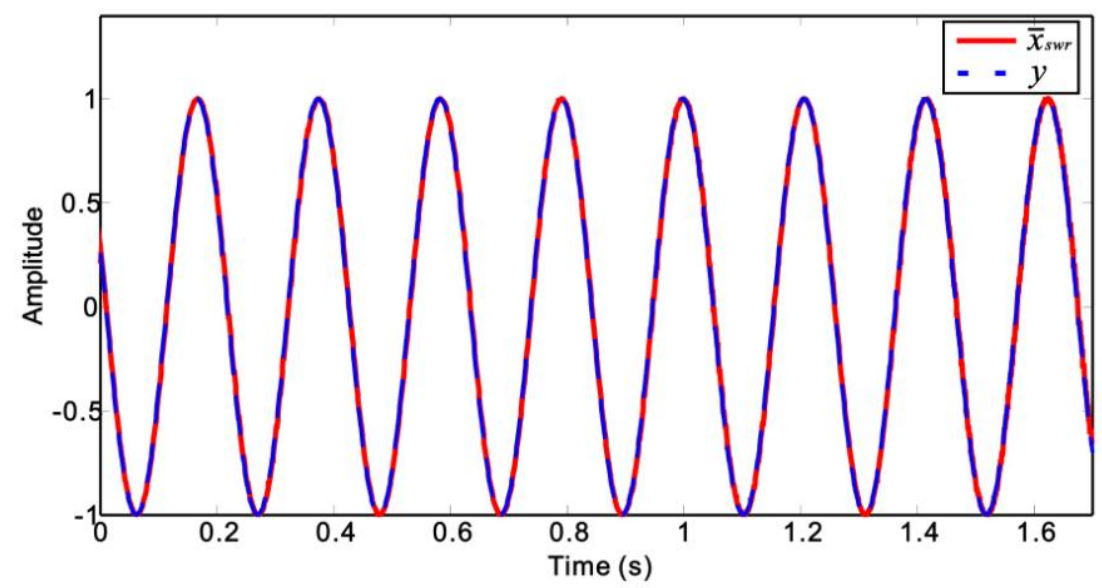

Fig. 8. The timescale of $\bar{x}_{s}(t)$ projected into the timescale of $y(t)$ after processing with warping and resampling algorithms.

$y_{w}(k)$ in Fig. 6, which now represents the number of data points instead of time.

To restore the length, $K$, of the warped signals back to the original length, $N$, a resampling algorithm is proposed in Fig. 7. This resampling algorithm also enables an 
optimal alignment of $\bar{x}_{s}(t)$ with the intact reference signal, $y(t)$, by resampling $\bar{x}_{s w}(k)$ as shown in Fig. 8, in which $\bar{x}_{s w r}(t)$ denoted the processed signal of $\bar{x}_{s w}(k)$ after the resampling.

Step 4: Warping and resampling of the original vibration measurement

As the reference signal, $y(t)$, is built using a constant rotational speed, $f_{s}$, it can be observed that the sequential application of the warping and resampling algorithms squeezes the time-dependent shaft rotational speed, $f_{s}(t)$, towards the constant rotational speed, $f_{s}$, in Fig. 8 through the optimal alignment of corresponding similar data points. Accordingly, this transformation procedure of the timescale also maps the time-varying frequency components, which are the harmonics of the shaft rotational speed, $f_{s}(t)$, into constant ones. This potentially makes the algorithm presented herein an effective approach for order spectrum analysis, the key of which lies in converting the instantaneous frequency trajectories of specified orders that change arbitrarily over time into lines parallel to the time axis on the time-frequency plane.

Motivated by this attribute, the original vibration signal, $x(t)$, is first warped based on the index, $i$, of the optimal alignment path, $W$, evaluated in Step 3. Thus, the warped signal of the original vibration measurement, $x_{w}(k)$, can be represented as:

$$
x_{w}(k)=x\left(w_{k}(1,1)\right)
$$

where the length of the warped signal, $x_{w}(k)$, equals the length of the warp path, $K$. Afterwards, the resulting time series, $x_{w}(k)$, is resampled based on the index, $j$, of the optimal alignment path, $W$, according to the pseudocode presented in Fig. 7. Through this procedure, the smeared spectral bands, the frequencies of which are fractional or integer multiples of $f_{s}(t)$, are squeezed into the respective individual peaks (the corresponding fractional or integer multiple of $f_{s}$ ). Assume the original vibration signal, $x(t)$, contains another signal, $x^{\prime}(t)$, whose frequency is a harmonic of the shaft rotational speed, $f_{s}(t)$, besides the extracted shaft-vibration signal, $x_{s}(t)$. The blue line in Fig. 9(a) shows the original spectrum of an arbitrarily selected harmonic signal 

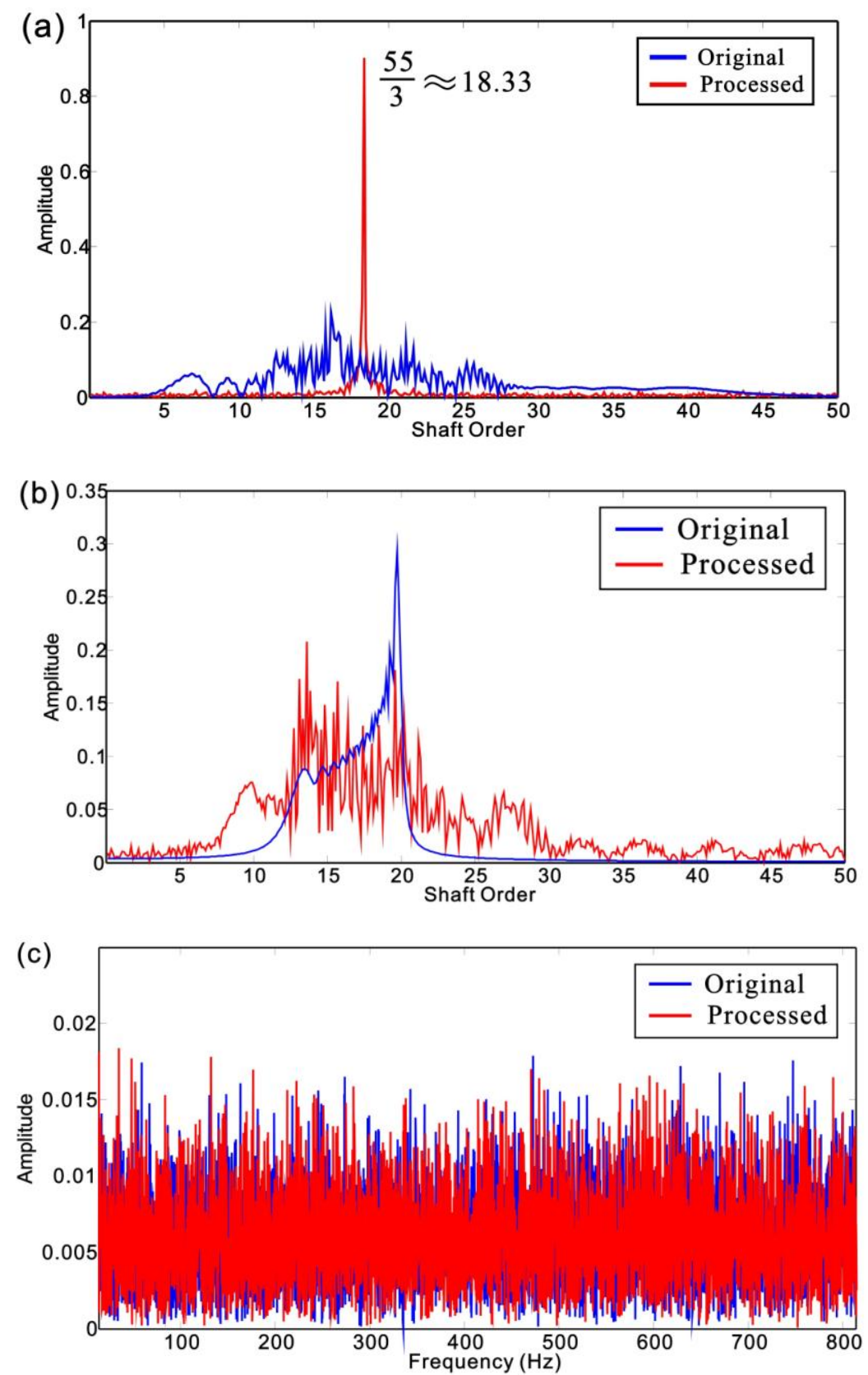

Fig. 9. The spectrum of (a) simulated $x^{\prime}(t)$ before and after the proposed technique, (b) simulated $x "(t)$ before and after the proposed technique, and (c) a white-noise series before and after the proposed technique.

$x^{\prime}(t)=\cos \left(2 \pi \frac{55}{3} f_{s}(t) t+\frac{3 \pi}{4}\right)$. The speed profile, $f_{s}(t)$, of this signal is the same as the speed profile used in $\bar{x}_{s}(t)$ and plotted in Fig. 5(a). The red line represents its processed spectrum after the sequential application of warping and resampling 
algorithms based on the optimal alignment path, $W$, between the $\bar{x}_{s}(t)$ and $y(t)$ evaluated in Step 3, which alleviates the spectral smearing and achieves a sharp peak at $\frac{55}{3} f_{s}\left(f_{s}=4.8 \mathrm{~Hz}\right)$

If the frequency of a smeared spectral band is nonproportional to $f_{s}(t)$, such as the vibration contribution from other mechanical components, its energy is not squeezed into certain frequency points after the proposed processing algorithm. This hypothesis is illustrated using an example signal $x^{\prime \prime}(t)=\cos \left(2 \pi \frac{55}{3}\left(5\left(1-e^{-(t+1)}\right)\right) t+\frac{3 \pi}{4}\right)$, as shown in Fig. 9(b). On the other hand, the proposed algorithm also does not squeeze the white noise into certain frequency points as demonstrated in Fig. 9(c), which presents the raw and processed spectrum of a simulated white-noise series. These traits of the algorithm imply that its application will not worsen SNR, and thus it is ideal for diagnosing the gearbox failures, in which the measured signals are usually contaminated by vibration from other mechanical components and background noise.

\section{Simulation-based validation of the presented algorithm}

This section discusses an analytical signal model for a single-stage, fixed-axis gearbox and demonstrates the diagnostic performance of the proposed approach through its simulations.

For the simulated case presented here, a single-stage, fixed-axis gear set with the number of pinion teeth represented by $N_{p}=10$ and the number of gear teeth represented by $N_{g}=15$ is assumed. The vibration response, $x(t)$, from this virtual gearbox consists of the shaft-vibration signal, $x_{s}(t)$, and gear-mesh vibration, $x_{m}(t)$. The dominant frequency components of $x_{s}$ are the gear-shaft rotational frequency, $f_{s}(t)$, and its harmonics, $n f_{s}(t)$. The important frequency components in $x_{m}$ are the fundamental gear-mesh harmonic, $f_{m}(t)\left(=N_{g} f_{s}(t)\right)$, its higher-order harmonics, $m f_{m}(t)$, and the sidebands around these components if a local gear fault is present. Thus, the overall vibration signal, $x(t)$, can be simulated as [12, 22-23]: 


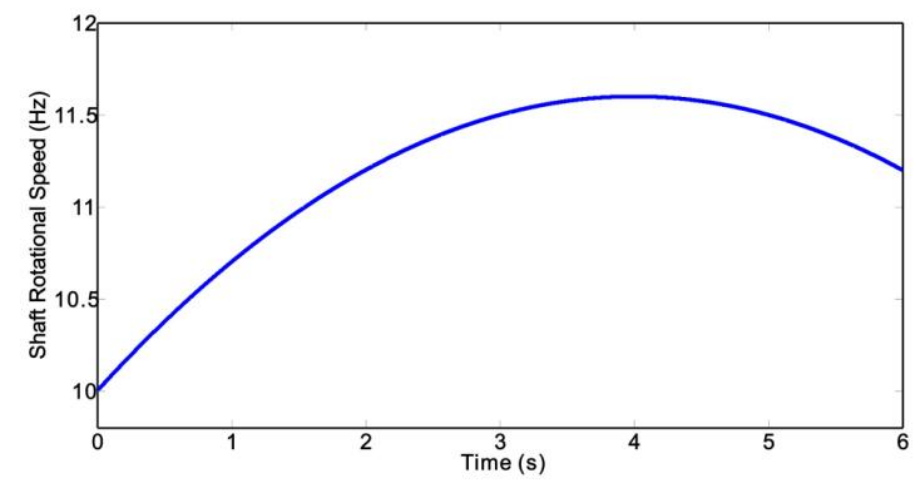

Fig. 10. Speed fluctuation profile, $f_{s}(t)$, used in the simulation.

$$
\begin{aligned}
x(t) & =x_{s}(t)+x_{m}(t) \\
& =\sum_{n=1}^{N} A_{s n}(t) \cos \left(2 \pi n f_{s}(t) t+\varphi_{n}\right) \\
& +\sum_{m=1}^{M} A_{m}(t)\left(1+a_{m}(t)\right) \cos \left(2 \pi m f_{m}(t) t+\phi_{m}+b_{m}(t)\right)
\end{aligned}
$$

where a nonlinear speed variation function, $f_{s}(t)=\left(1+0.08 t-0.08 t^{2}\right) f_{s}$, is used to simulate a speed fluctuation of up to $15 \%$ around the nominal shaft rotational frequency $f_{s}=10 \mathrm{~Hz}$. Figure 10 plots the nonlinear fluctuating speed profile, $f_{s}(t)$. The amplitude and phase modulation (AM \& PM) functions, $a_{m}(t)$ and $b_{m}(t)$, due to a local fault at a gear tooth are expressed as [24]:

$$
\begin{gathered}
a_{m}(t)=\sum_{l=1}^{L} a_{m l} \cos \left(2 \pi l f_{s}(t) t+\delta_{m l}\right) \\
b_{m}(t)=\sum_{l=1}^{L} b_{m l} \cos \left(2 \pi l f_{s}(t) t+\beta_{m l}\right)
\end{gathered}
$$

If the gearbox is free of any defect, the amplitudes of Eqs. (11) and (12) are equal to 0. For the simplicity of the simulations, only the first shaft order, $n=1$, and the fundamental gear-mesh harmonic, $m=1$, are considered, and their initial phase, $\varphi_{1}$, and $\phi_{1}$ are set as $2 \pi / 3$ and $\pi / 4$, respectively. A time-dependent function, $A s_{1}(t)=1+$ $0.5 t-0.05 t^{2}$, is assumed as the amplitude of the shaft vibration signal, $x_{s}(t)$, to simulate the effect of the nonstationary external load. Afterwards, it is assumed that the amplitude of the fundamental gear-mesh harmonic is $A_{1}(t)=3 A s_{1}(t)$. Then, the harmonic order, $l$, of the modulation function, $a_{m}(t)$ and $b_{m}(t)$, is set to 3 , in which amplitudes $a_{11}=0.29, a_{12}=0.25, a_{13}=0.2 ; b_{11}=0.07, b_{12}=0.1, b_{13}=-0.03$ and $\delta_{11}=$ 

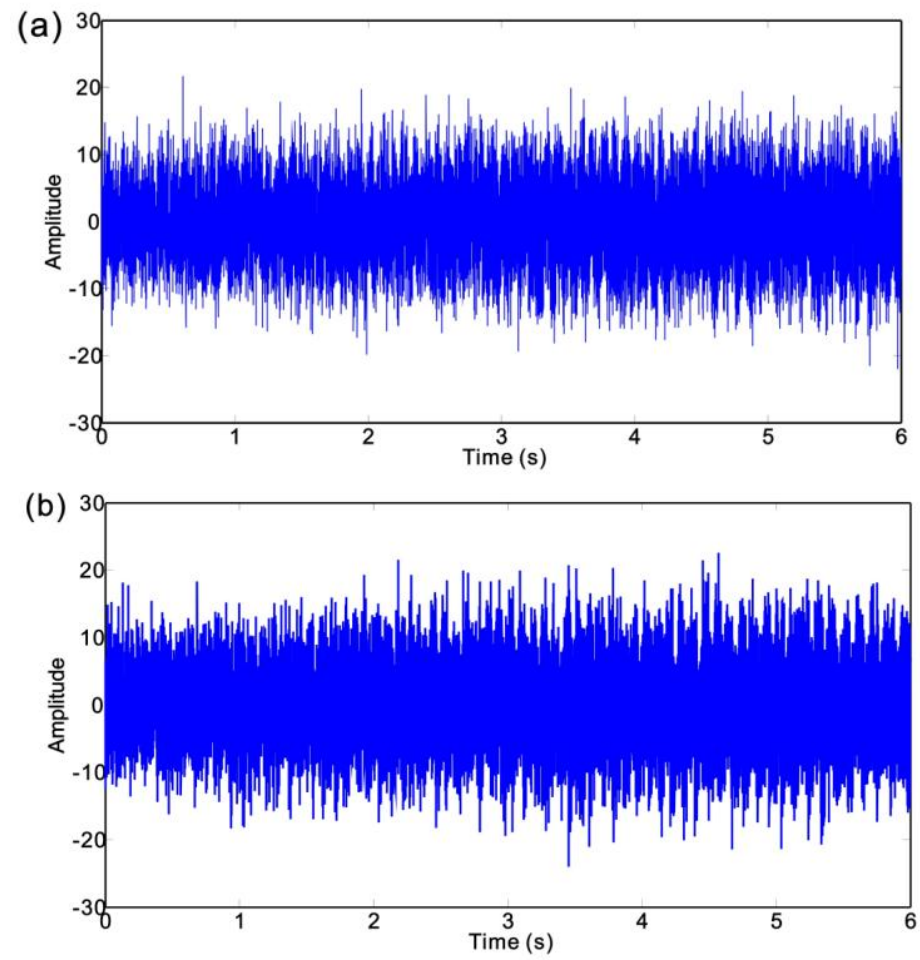

Fig. 11. The waveform of (a) the fixed-axis gearbox signal under a healthy condition, and (b) the fixed-axis gearbox signal under a gear fault condition.
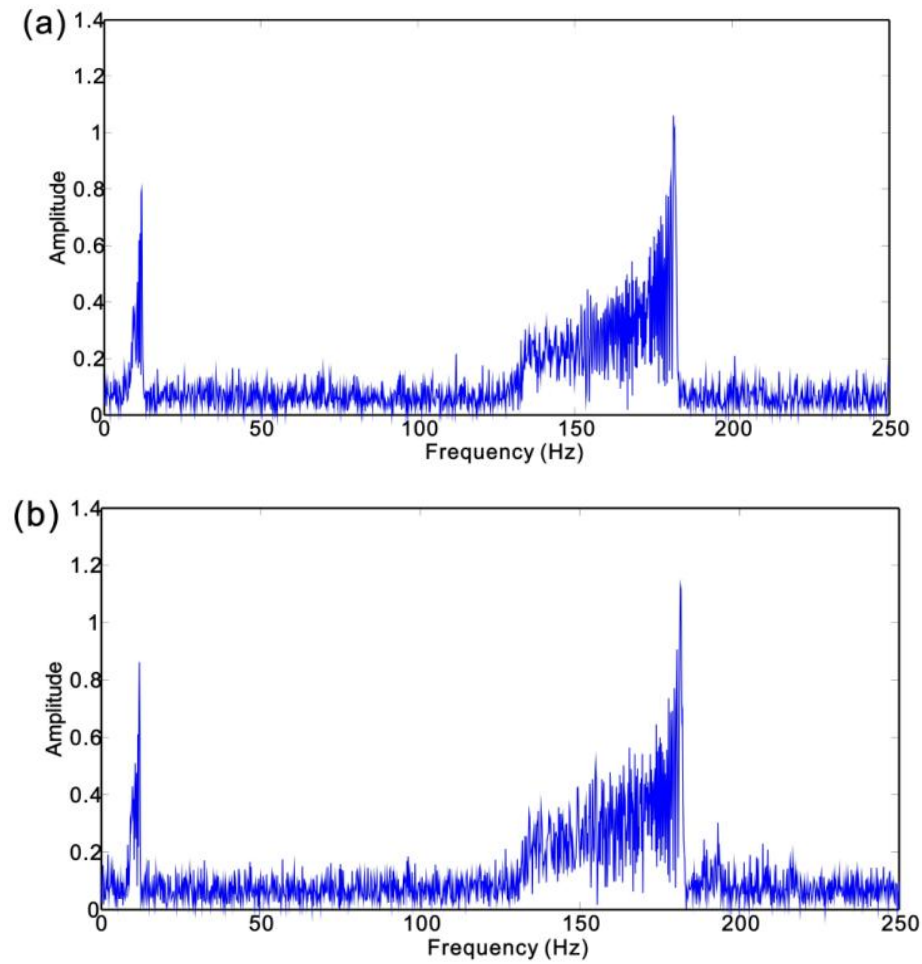

Fig. 12. The spectrum of (a) the fixed-axis gearbox signal under a healthy condition, and (b) the fixed-axis gearbox signal under a gear fault condition.

$\pi / 5, \delta_{12}=-\pi / 6, \delta_{13}=2 \pi / 5 ; \beta_{11}=-\pi / 2, \beta_{12}=\pi / 3, \beta_{13}=2 \pi / 3$ are arbitrarily chosen to 
(a)
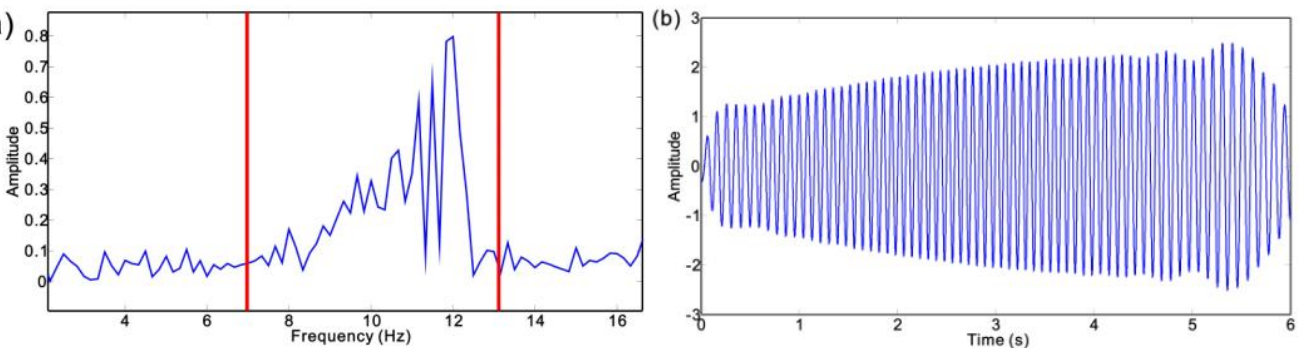

Fig. 13. (a) The detailed spectrum around the shaft rotational frequency, $f_{s}(t)$, and (b) the filtered shaft-vibration signal, $x_{s}(t)$.

simulate a faulty gear. Gaussian white noise with $\mathrm{SNR}=0 \mathrm{~dB}$ is also added to the signal, $x(t)$, to simulate the measured noise in a practical system. The sampling
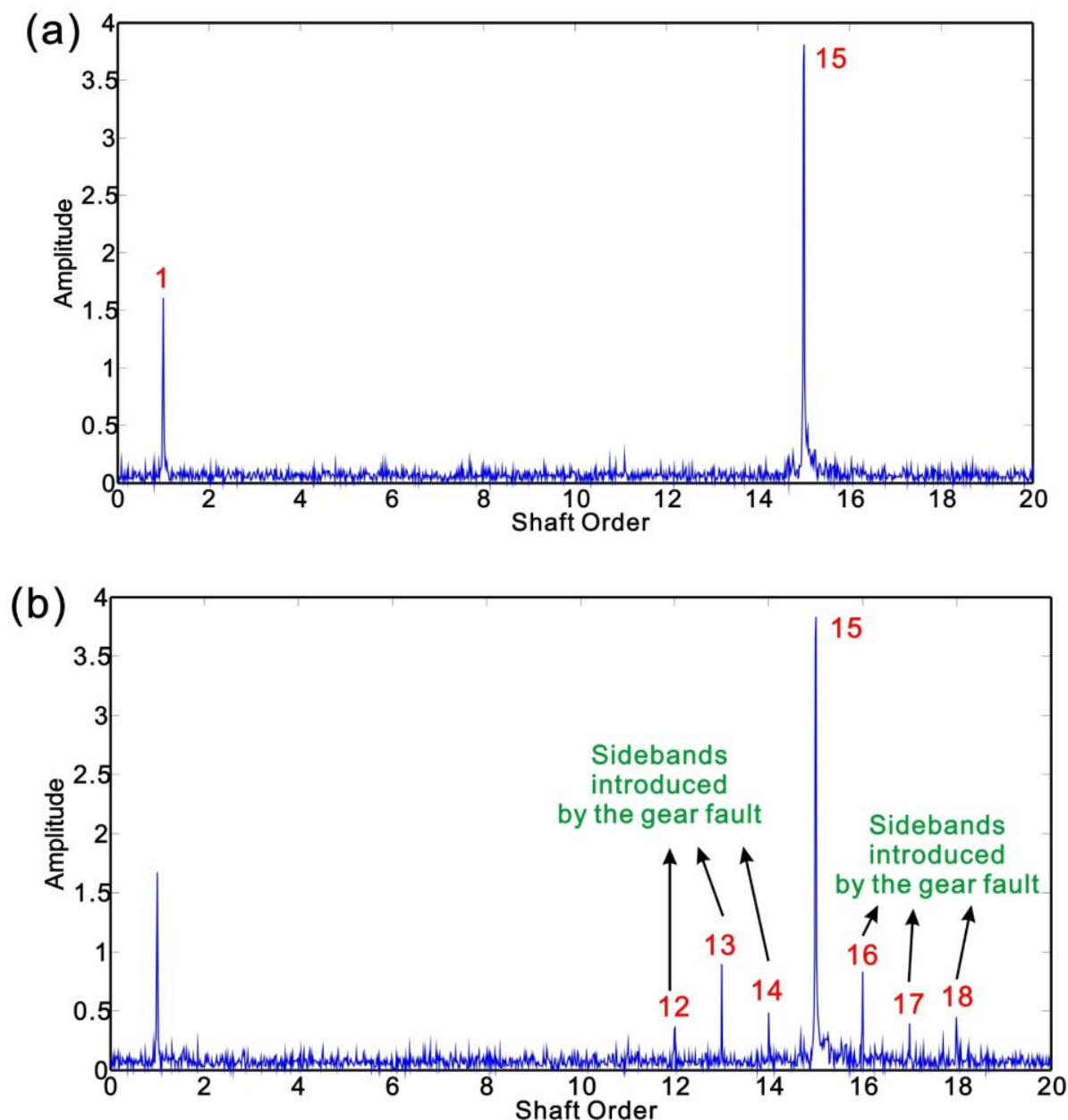

Fig. 14. The spectrum of (a) the fixed-axis gearbox signal under a healthy condition after the proposed algorithm, and (b) the fixed-axis gearbox signal under a gear fault condition after the proposed algorithm. 
interval used in this simulation is $4 \times 10^{-4}$ seconds $(2.5 \mathrm{kHz})$. The time-domain waveforms of the simulated healthy and faulty cases are plotted in Fig. 11, which indicates that the modulation features caused by the gear fault are mostly concealed by noise. The spectra of the simulated signals are shown in Fig. 12(a) and (b). However, the fault features, which are the multicomponent AM and PM signals, cannot be distinguished from these spectral components because of the smearing phenomenon caused by the speed fluctuations. Afterwards, the proposed tacho-less fault-diagnostic algorithm described in Section 3 is applied to the simulated signals. Figure 13(a) presents the detailed spectrum around the nominal gear-shaft rotational frequency, $f_{s}$. Visually, the pass band of the filter can be set between $7.5 \mathrm{~Hz}$ and $13 \mathrm{~Hz}$ to include the entire energy content of the fundamental shaft rotational harmonic. The filtered signal is shown in Fig. 13(b), which is then used as the input signal, $x_{s}(t)$, for the proposed scheme described in Section 3. Figure 14 presents the spectra of the
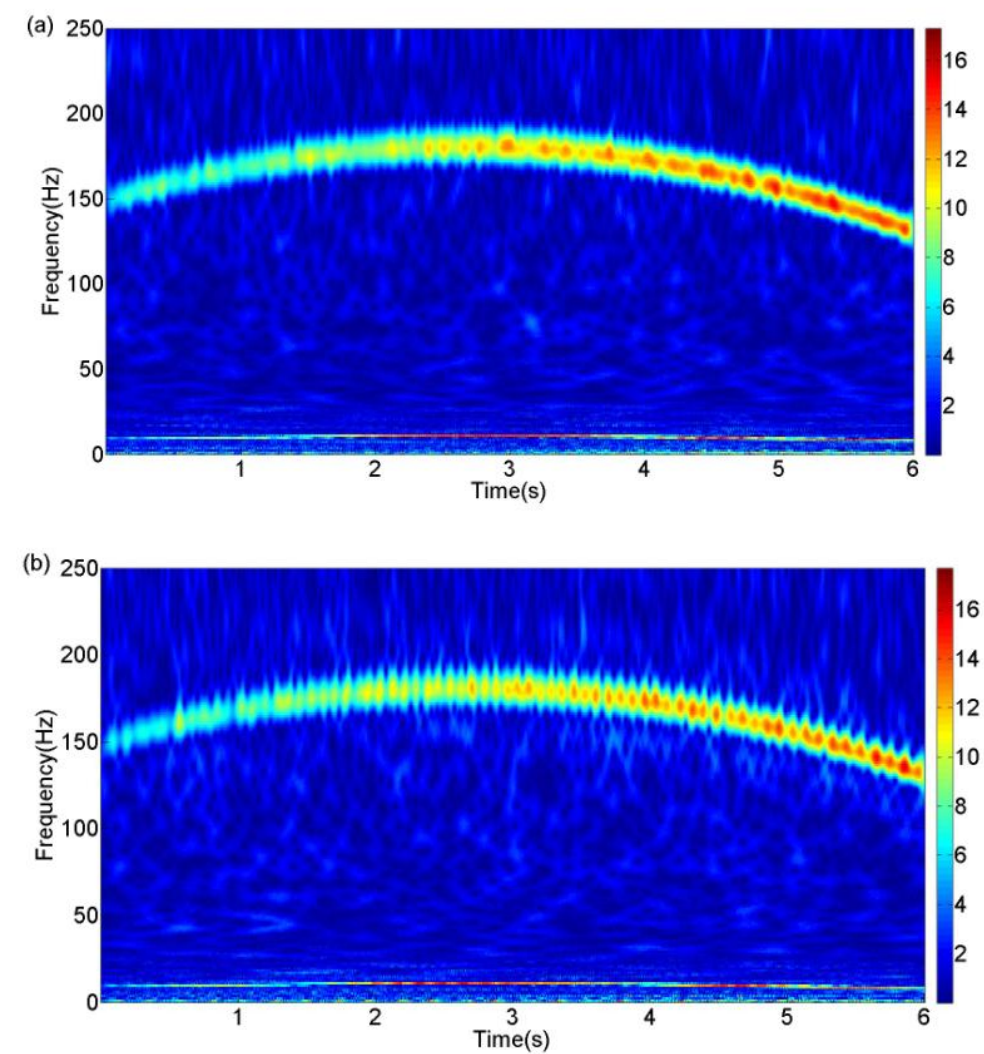

Fig. 15. The wavelet transform of (a) the fixed-axis gearbox signal under a healthy condition, and (b) the fixed-axis gearbox signal under a gear fault condition. 
simulated signals for healthy and faulty cases processed with the proposed diagnostic scheme. It can be observed that the severe spectral smearing caused by the speed fluctuations in Fig. 12 is alleviated from the processed spectra and the sidebands introduced by the gear fault can be identified clearly as a result of the proposed diagnostic algorithm. Thus, the simulations validate the capability of the proposed tacho-less algorithm to identify gear defects. Figure 15 presents the time-frequency results of the original signals by the Morlet wavelet transform. The fault features are barely observed in Fig. 15. This phenomenon is because the time-frequency transform cannot achieve enough time and frequency resolution simultaneously because of the uncertainty principle of the time-frequency representation.

\section{Experimental verification}

5.1. Wind turbine gearbox test rig at the National Renewable Energy Laboratory

In this section, the proposed tacho-less diagnostic algorithm is experimentally validated through measurements from a $750-\mathrm{kW}$ industrial wind turbine gearbox. The gearbox was tested in a 2.5-MW dynamometer test facility at the National Wind Technology Center at the National Renewable Energy Laboratory (NREL). This facility is capable of providing static, highly accelerated life and model-in-the-loop testing. The internal view of the tested wind turbine gearbox is presented in Fig. 16.

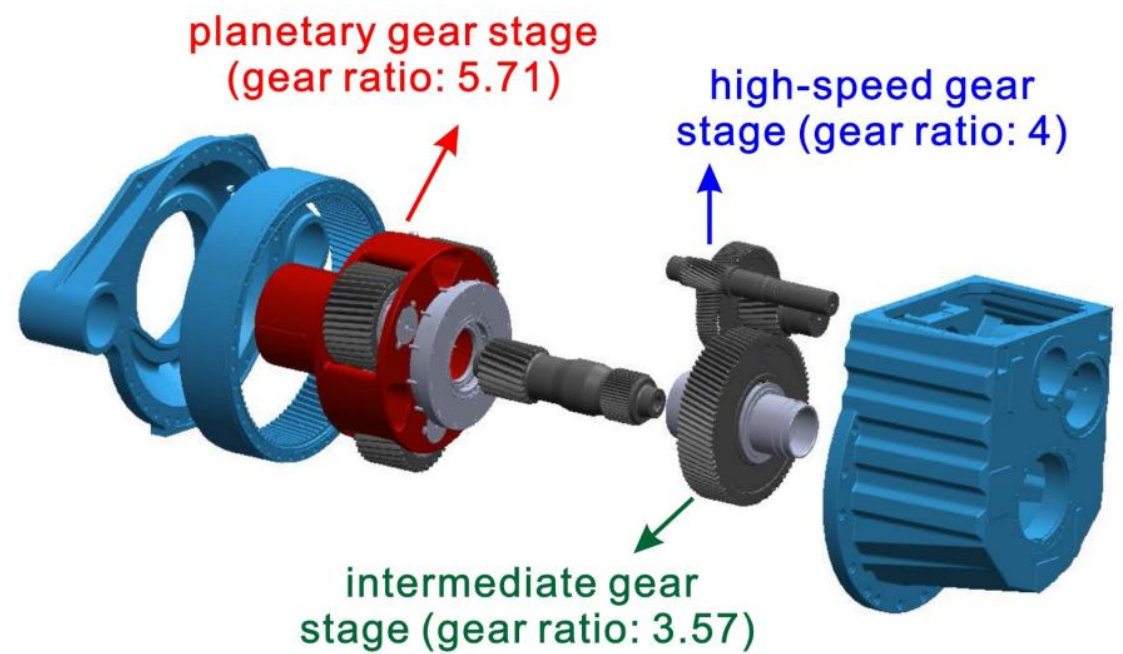

Fig. 16. The internal view of the planetary wind turbine gearbox (Credit: NREL). 
(a)

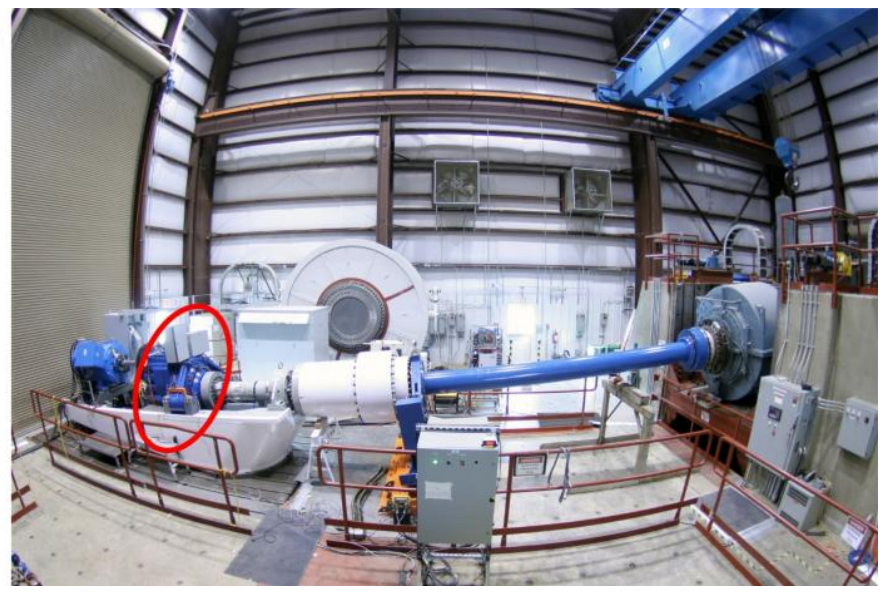

(b)

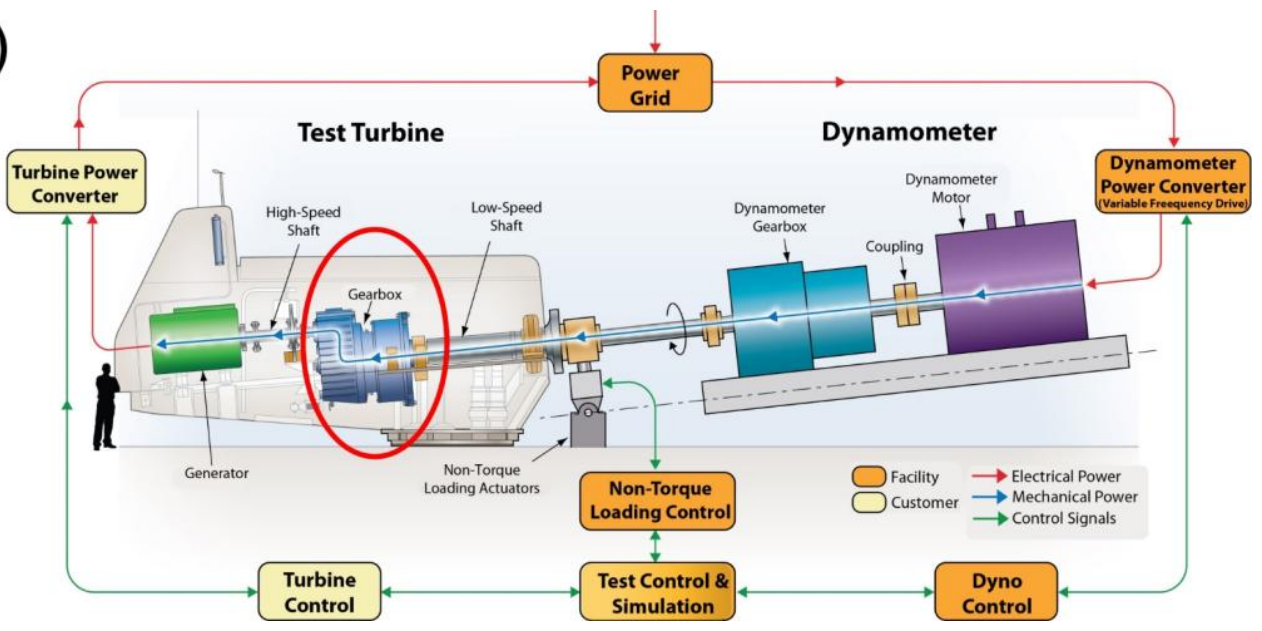

(c) AN4

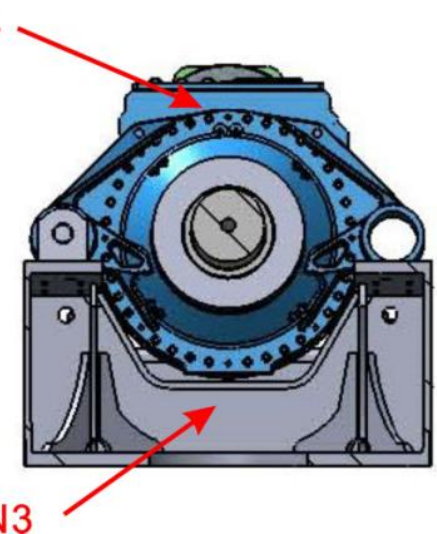

Fig. 17. (a) The 750-kW wind turbine gearbox on NREL's $2.5-\mathrm{MW}$ dynamometer (Credit: Lee Jay Fingersh/NREL 16913), (b) diagram of the dynamometer test rig (Credit: NREL), and (c) two accelerometers, AN3 and AN4, mounted on the annulus gear of the planetary gear set radial at 6 and 12 o'clock.

This gearbox consists of a planetary gear stage followed by two parallel-axis gear 
stages - the intermediate stage and the high-speed stage (number of teeth on intermediate gear $N_{i g}=82$, number of teeth on intermediate pinion $N_{i p}=23$, number of teeth on high-speed gear $N_{h g}=88$, and number of teeth on high-speed pinion $N_{h p}=$ 22). The input side of the gearbox is a low-speed shaft connected to the carrier of an equally spaced three-planet gear set (number of teeth on annulus gear $N_{a}=99$, number of teeth on sun gear $N_{s}=21$, and number of teeth on planet pinion $N_{p}=39$ ) of the gearbox and the sun gear is connected with the intermediate parallel gear stage. Thus, the overall gear ratio of the gearbox equals $\left(1+N_{a} / N_{s}\right) \times\left(N_{i g} / N_{i p}\right) \times\left(N_{h g} / N_{h p}\right)=5.71$ $\times 3.57 \times 4=81.49$. This industrial gearbox was damaged when installed on a turbine at the Ponnequin wind farm in Colorado during its operation. It was initially installed in the turbine and put into unattended operation on September 14, 2009. However, after the bearing temperature exceeded the threshold and reports of oil loss from the gearbox, which resulted in damage to its internal bearings and gears, the turbine was stopped on October 5, 2009. Subsequently, the gearbox was removed from the turbine to avoid potential catastrophic failure. Because the damage was not catastrophic, the gearbox was shipped to NREL and tested in their 2.5-MW dynamometer facility before being disassembled. Measurements obtained from two accelerometers, AN3 and AN4 mounted on the annulus gear (Fig. 17), were used in this work for the diagnosis of the planetary gear set. The vibration signal is measured at $25 \%$ of rated

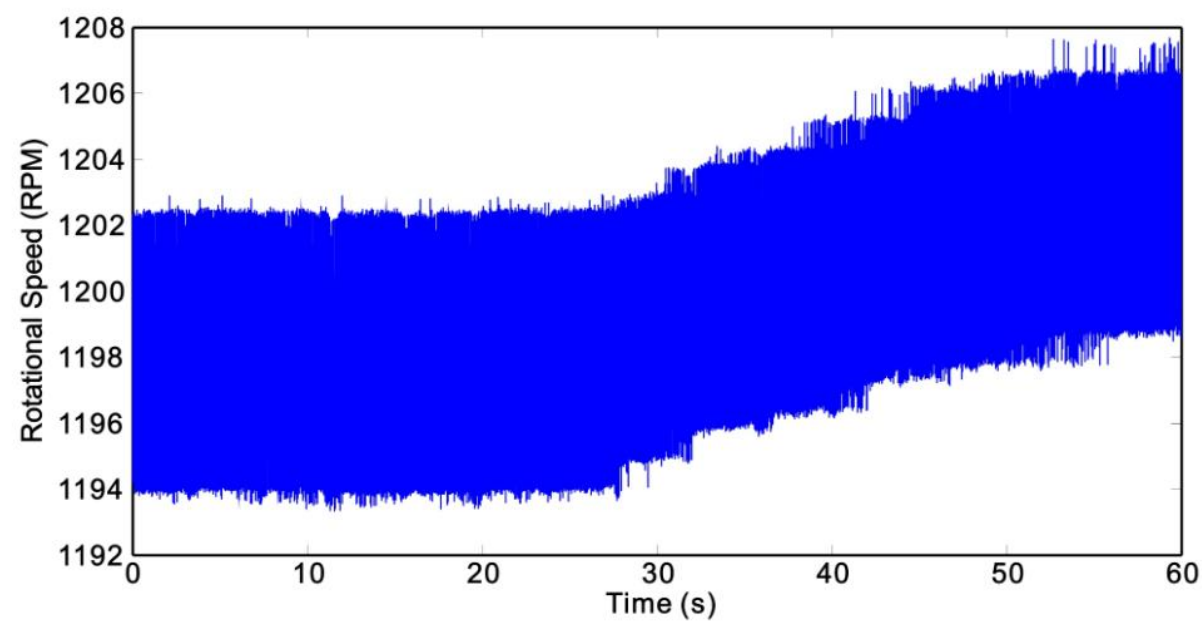

Fig. 18. The measured speed profile of the high-speed shaft fluctuating around $1 \%$ in $60 \mathrm{~s}$. 
(a)

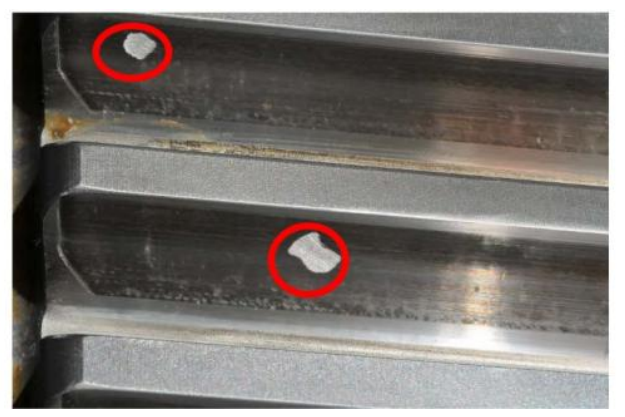

(b)

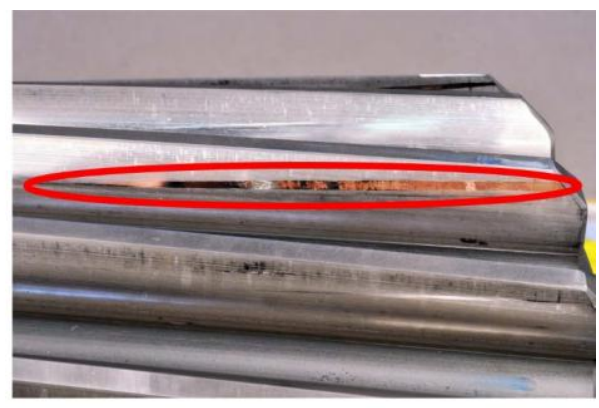

Fig. 19. (a) Moderate gear scuffing and polishing wear on the annulus gear (Credit: GEARTECH, NREL 19750), and (b) severe fretting corrosions on the sun pinion (Credit: GEARTECH, NREL 19749).

power and nominal angular speed 1,200 rpm $\left(f_{s}=20 \mathrm{~Hz}\right)$ of the high-speed shaft. This means that the nominal frequency of the carrier $f_{c}$ equals $0.25 \mathrm{~Hz}\left(\frac{f_{s}}{81.49}\right)$, which mimics the slow rotational speed of the rotor in practical wind turbines. The nominal gear-mesh frequency under such conditions of the planetary gear set, $f_{m}$, is $24.3 \mathrm{~Hz}\left(f_{c}\right.$ $\times N_{a}$ ). The sampling rate for measurement was $40 \mathrm{kHz}$, which is much higher than the gear-mesh harmonics of the planetary gear set. Therefore, the measured data was first down-sampled to $2,000 \mathrm{~Hz}$ to speed up the computational time. Despite controlled conditions, around $1 \%$ fluctuations in the operational speed can be observed in the speed profile of the high-speed shaft as measured by the tachometer and shown in Fig. 18. This signal from the tachometer can also be observed to be contaminated with the high-frequency, ambient, electromagnetic noise. It is noteworthy, because the tachometer signal is not used to process the experimental data in this paper, but such noisy speed measurements can challenge the tacho-based order-tracking approach that may be used to alleviate the spectral smearing. After the dynamometer test, the gearbox was sent to a rebuild shop, where it was disassembled and a detailed inspection $[25,26]$ was conducted. This detailed inspection indicated damage to the annulus gear and the sun gear of the planetary gear set. Severe fretting corrosions were observed on the sun pinion and moderate gear scuffing and polishing wear on the annulus gear (Fig. 19).

5.2. Distinct vibration sideband characteristics of the planetary gear system 
Because a planetary gear set employs $P$ number of identical planet pinions attached to a carrier that revolve around the sun gear, the vibration signals picked up by a fixed sensor attached to planetary-gearbox housing are influenced by the AM phenomenon even under the healthy condition as described in [27, 28]. Thus, the vibration spectrum of the planetary gearbox from a fixed accelerometer exhibit complicated and distinct sideband characteristics [29]. Such sideband behaviors are summarized in [30]. If the planetary gear set has a damaged annulus gear tooth, additional sidebands will appear at $m f_{m}+q f_{c}$ (equal to $\left(m N_{a}+q\right) f_{c}, m$ and $q$ are integer numbers) only when $m N_{a}+q=$ an integer multiple of $P$. If there is a local fault on the sun gear, it can be indicated by sidebands at $\left(m N_{a}+q \frac{N_{a}}{N_{s}}+k\right) f_{c}$ only when $m N_{s}+q-k=$ an integer multiple of $P$. The potential frequency locations indicating the existence of an annulus gear fault are highlighted as green lines in the carrier order spectra for the tested gearbox as shown in Fig. 20. For example, the frequency components that are integer multiples of the number of planets $(P=3)$ and away from the planetary gear-mesh frequency of the $99^{\text {th }}$ carrier order, imply the annulus gear fault, such as $87^{\text {th }}, 90^{\text {th }}$, $114^{\text {th }}, 117^{\text {th }}$, and $120^{\text {th }}$ carrier orders. Similarly, the potential frequency locations implying the existence of a sun gear fault are highlighted as red lines, such as $87.57\left(=99-2 \times \frac{99}{21}-2\right), 104.7\left(=99+\frac{99}{21}+1\right)$ and $110.4\left(=99+2 \times \frac{99}{21}+2\right)$ times the carrier frequency. Additionally, the locations $\left(\omega_{m} \pm q \omega_{c}\right.$, with $q$ being an integer number) highlighted by pink lines around the fundamental gear-mesh frequency suggest an issue with the position shift of planets or the unequal load sharing among the planets $[31,32]$.

\subsection{Signal analysis}

To conduct the vibration-based diagnosis, the carrier order spectrum around the fundamental planetary gear-mesh frequency measured by AN3 is plotted in Fig. 20(a). It can be observed that the frequency components are blurred and difficult to analyze even in the presence of small speed fluctuations under this controlled experimental environment. Afterwards, the proposed tacho-less diagnostic procedure described in 

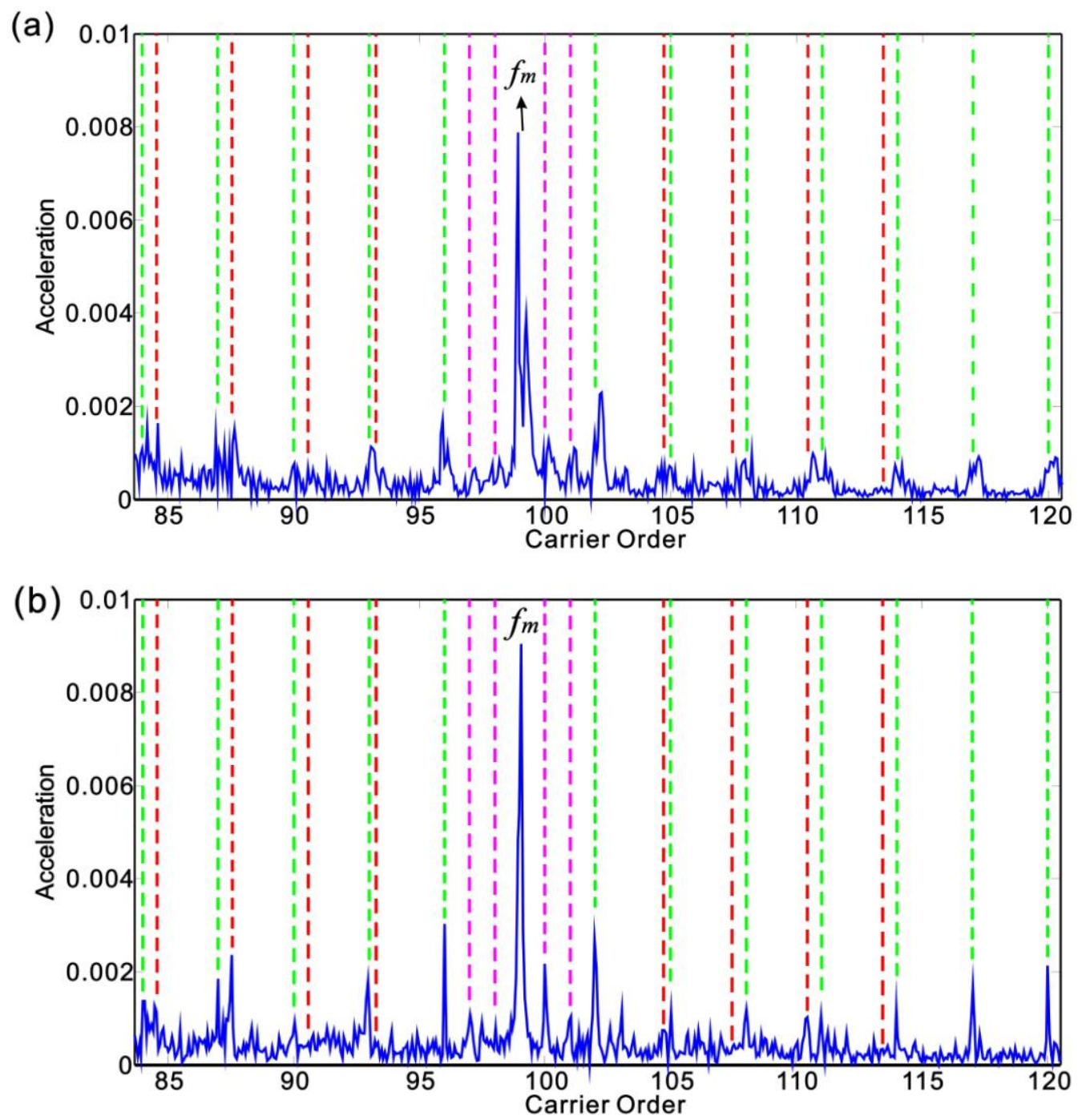

Fig. 20. (a) The original measured vibration spectrum around the fundamental gear-mesh frequency of the planetary gear set, and (b) the vibration spectrum after the proposed algorithm around the fundamental gear-mesh frequency of the planetary gear set.

Section 3 was applied to process the measured vibration signal from AN3. The central frequency of the band-pass filter was set as $20 \mathrm{~Hz}$ to extract the vibration signal of the high-speed shaft. As discussed in the beginning of this section, the actual carrier rotational frequency, $f_{c}(t)$, and gear-mesh frequency of the planetary gear set, $f_{m}(t)$, are both proportional to the rotational frequency of the high-speed shaft, $f_{s}(t)$. Furthermore, because of the small speed fluctuations and a large number of frequency components arising from the multistage gear sets, a narrow bandwidth of $1 \mathrm{~Hz}$ is chosen for the band-pass filter. The effectiveness of the proposed algorithm for fault 
detection from practical measured signals is demonstrated in Fig. 20(b). It can be deduced that the proposed approach is capable of alleviating the spectral smearing caused by speed fluctuations, which squeezes the energy more narrowly to make the fault signatures in annulus and sun gear easier to identify. These highlighted components clearly show lesser smearing and sharper peaks in Fig. 20(b) as compared to Fig. 20(a). Thus, the proposed tacho-less diagnostic algorithm has the potential to assist in the identification of fault information from gearbox vibration signals under speed fluctuations without any rotational speed measurement.

\section{Conclusions}

Operation of gearboxes in industrial environments usually exhibits fluctuations in speed and load around nominal operating conditions. The conventional spectral analysis has a limitation in such cases because of the spectral smearing, which makes the identification and extraction of the fault signature difficult. Order-tracking techniques using speed measurement can alleviate the smearing effect; however, the required additional sensors are often lacking in the industrial environment, and industrial-grade speed sensors can often introduce significant sensor noise-challenging its application. To avoid taking speed measurements, some tacho-less diagnostic techniques exist, which estimate the instantaneous shaft angular position/speed and employ sophisticated equi-angular resampling algorithms to resample the original data into the angular domain that may introduce extra decimation and/or interpolation errors. However, unlike the idea of the previous published tacho-less order-tracking techniques, the proposed tacho-less fault signature enhancement algorithm projects the original timescale to a new transformed timescale by warping the measured vibration signals, in which the time-varying speed of the shaft is squeezed towards a constant reference speed. Consequently, on this transformed timescale, the smeared spectral bands, the frequencies of which are proportional to the time-varying speed of the shaft, are also squeezed into respective individual peaks. The presented diagnostic method requires only an approximate knowledge of the operational speed besides the measured gearbox vibration signal. 
The performance and applicability of the proposed approach is demonstrated both by the analytical simulation of a fixed-axis gearbox under the nonstationary conditions and experimental data measured from an industrial wind turbine planetary gearbox that was damaged during operation. Both the simulation and experimental results indicate that the proposed approach is capable of alleviating the spectral smearing of the vibration spectrum by squeezing the energy towards dominant frequency features that are harmonics of the fundamental shaft-rotational frequency. Thus, the tacho-less diagnostic approach described here advances the state of the art of gearbox diagnostic techniques without requiring the rotational speed measurement and has the potential to identify faults from gearbox vibration signals for practical applications under realistic industrial environments.

\section{Acknowledgements}

This research is supported by National Natural Science Foundation of China (Grant No. 51605349) and Fundamental Research Funds for the Central Universities of China (WUT: 163104002). The experimental work presented in this article was supported by the U.S. Department of Energy under Contract No. DE-AC36-08GO28308 with the National Renewable Energy Laboratory. Funding for the experimental work was provided by the DOE Office of Energy Efficiency and Renewable Energy, Wind and Water Energy Technologies Office.

The U.S. Government retains and the publisher, by accepting the article for publication, acknowledges that the U.S. Government retains a nonexclusive, paid-up, irrevocable, worldwide license to publish or reproduce the published form of this work, or allow others to do so, for U.S. Government purposes.

\section{References}

[1] Rui Man, Yushu Chen, Qingjie Cao, Research on dynamics and fault mechanism of spur gear pair with spalling defect, Journal of Sound and Vibration 331(2012) 2097-2109.

[2] Guolin He, Kang Ding, Huibin Lin, Gearbox coupling modulation separation 
method based on match pursuit and correlation filtering, Mechanical Systems and Signal Processing 25(2016) 597-611.

[3] J. Antoni, F. Bonnardot, A. Raada, M. El Badaoui, Cyclostationary modelling of rotating machine vibration signals, Mechanical Systems and Signal Processing 18(2004) 1285-1314.

[4] A. Jablonski, T. Barszcz, Validation of vibration measurements for heavy duty machinery diagnostics, Mechanical Systems and Signal Processing 38(2013) $248-263$.

[5] L. F. Villa, A. Renones, J. R. Peran, L. J. De Miguel, Angular resampling for vibration analysis in wind turbines under non-linear speed fluctuation, Mechanical Systems and Signal Processing 25(2011) 2157-2168.

[6] H. Xiao, X. Zhou, Y. Shao, Application of an improved dynamic time synchronous averaging method for fault diagnosis in conditions of speed fluctuation and no tachometer, Proceedings of the Institution of Mechanical Engineers, Part C: Journal of Mechanical Engineering Science, 20150954406215597956.

[7] F. Bonnardot, M. El Badaoui, R. B. Randall, J. Daniere, F. Guillet, Use of the acceleration signal of a gearbox in order to perform angular resampling (with limited speed fluctuation), Mechanical Systems and Signal Processing 19(2005) 766-785.

[8] F. Cobmet, L. Gelman, An automated methodology for performing time synchronous averaging of a gearbox signal without speed sensor, Mechanical Systems and Signal Processing 21(2007) 2590-2606.

[9] F. Comber, R. Zimroz, A new method for the estimation of the instantaneous speed relative fluctuation in a vibration signal based on the short time scale transform, Mechanical Systems and Signal Processing 23(2009) 1382-1397.

[10] Chuan Li, Ming Liang, Time-frequency signal analysis for gearbox fault diagnosis using a generalized synchrosqueezing transform, Mechanical Systems and Signal Processing 26(2012) 205-217.

[11] R. Uma Maheswari, R. Umamaheswari, Trends in non-stationary signal processing techniques applied to vibration analysis of wind turbine drive train - A contemporary survey, Mechanical Systems and Signal Processing 85(2017) 296-311.

[12] Guolin He, Kang Ding, Weihua Li, Xintao Jiao, A novel order tracking method for wind turbine planetary gearbox vibration analysis based on discrete spectrum correction technique, Renewable Energy 87(2016) 364-375.

[13] M. Inalpolat, A. Karaman, A theoretical and experiment investigation of modulation sidebands of planetary gear sets, Journal of Sound and Vibration 323(2009) 677-696.

[14] Murat Inalpolat, Ahmet Karaman, A dynamic model to predict modulation sidebands of a planetary gear set having manufacturing errors, Journal of Sound and Vibration 329(2010) 371-393.

[15] Eamonn Keogh, Chotirat Ann Ratanamahatana, Exact indexing of dynamic time warping, Knowledge and Information Systems 7(2005) 358-386.

[16] D. Zhen, T. Wang, F. Gu, A. D. Ball, Fault diagnosis of motor drives using stator current signal analysis based on dynamic time warping, Mechanical Systems and Signal Processing 34(2013) 191-202. 
[17] S. Salvador, P. Chan, Toward accurate dynamic time warping in linear time and space, Intelligent Data Analysis 11(2007) 561-580.

[18] Liu Hong. Jaspreet S. Dhupia, A time domain approach to diagnose gearbox fault based on measured vibration signals, Journal of Sound and Vibration 333(2014) 2164-2180.

[19] Liu Hong, Jaspreet S. Dhupia, A time-domain fault detection method based on an electrical machine stator current measurement for planetary gear-sets, presented at 2013 IEEE/ASME International Conference on Advanced Intelligent Mechatronics, 2013/7/9-2013/7/12, Wollongong, NSW.

[20] M. Ch. Pan, Y. F. Lin, Further exploration of Vold-Kalman-filtering order tracking with shaft-speed information-I: Theoretical part, numerical implementation and parameter investigations, Mechanical Systems and Signal Processing 20(2006) 1134-1154.

[21] M. Zhao, J. Lin, X. Wang, Y. Lei, J. Cao, A tacho-less order tracking technique for large speed variations, Mechanical Systems and Signal Processing 40(2013) 76-90.

[22] Liu Hong, Yongzhi Qu, Jaspreet S. Dhupia, Y. Tan, A novel fault diagnostic technique for gearboxes under speed fluctuations without angular speed measurement, presented at ASME/ISCIE 2016 International Symposium on Flexible Automation, 2016/9/1-2016/8/3, Cleveland, Ohio.

[23] Wilson Q. Wang, Fathy Ismail, M. Farid Golnaraghi, Assessment of gear damage monitoring techniques using vibration measurements, Mechanical Systems and Signal Processing 15(2001) 905-922.

[24] Zhihong Man, Wenyi Wang, Suiyang Khoo, Juliang Yin, Optimal sinusoidal modelling of gear-mesh vibration signals for gear diagnosis and prognosis, Mechanical Systems and Signal Processing 33(2012) 256-274.

[25] R. Errichello, J. Muller, Gearbox Reliability Collaborative Gearbox 1 Failure Analysis Report, NREL/SR-5000-53062(2012).

[26] Shuangwen Sheng, Wind turbine gearbox condition monitoring round robin study — vibration analysis, NREL/TP-5000-54530(2012).

[27] J. McNames, Fourier series analysis of epicyclic gearbox vibration, ASME Journal of Vibration and Acoustics 124(2002) 150-152.

[28] Bin Zhang, Khawaja T., Patrick R., Vachtsevanos G., Application of blind deconvolution de-noising in failure prognosis, IEEE Transactions of Instrumentation and Measurement 58(2009) 303-310.

[29] Zhipeng Feng, Xiaowang Chen, Ming Liang, Joint envelope and frequency order spectrum analysis based on iterative generalized demodulation for planetary gearbox fault diagnosis under nonstationary conditions, Mechanical Systems and Signal Processing 76-77(2016) 242-264.

[30] Liu Hong, Jaspreet S. Dhupia, Shuangwen Sheng, An explanation of frequency features enabling detection of faults in equally-spaced planetary gearbox, Mechanism and Machine Theory 73(2014) 169-183.

[31] W. D. Mark, J. A. Hines, Stationary transducer response to planetary-gear vibration excitation with non-uniform planet loading, Mechanical Systems and Signal 
Processing 23(2009) 1366-1381.

[32] Yaguo Lei, Zongyao Liu, Jing Lin, Fanbo Lu, Phenomenological models of vibration signals for condition monitoring and fault diagnosis of epicyclic gearboxes, Journal of Sound and Vibration 369(2016) 266-281. 